\title{
THE GEOMETRICAL CONSTRUCTIONS LIFTING TENSOR FIELDS OF TYPE $(0,2)$ ON MANIFOLDS TO THE BUNDLES OF A-VELOCITIES
}

\author{
W. M. MIKULSKI
}

\section{Introduction}

Let $A$ be a Weil algebra. The fibre bundle $T^{A} M$ of $A$-velocities over a manifold $M$ was described by A. Morimoto [15] as another description of the bundle of near $A$-points by Weil [17]. In [4] for any tensor field $\tau$ of type $(0,2)$ on $M$ and any functional $\lambda \in A^{*}$ we have defined the so called $\lambda$-lift of $\tau$ to $T^{A} M$. We recall this construction in Example 1.3. The $\lambda$-lift of $\tau$ is a naturally induced tensor field of type $(0,2)$ on $T^{A} M$.

In this paper we study the problem how a tensor field of type $(0,2)$ on $M$ can induce tensor fields of types $(0,1)$ and $(0,2)$ on $T^{A} M$. In Section 1 we present some constructions of such type. Some new lifts of tensor fields of type $(0,2)$ to $T^{A} M$ are presented. In Section 2 we remark that the idea of such constructions is reflected in the concept of natural operators $T^{(0,2)} \rightarrow T^{(0,1)} T^{A}$ and $T^{(0,2)} \rightarrow T^{(0,2)} T^{A}$, cf. [6]. The rest of the paper is dedicated to the proof of the following classification theorems.

Theorem 0.1. Let $A$ be a Weil algebra with $p$ variables. For $n$-manifolds ( $n \geq$ $p+2)$, the space of all natural operators $T^{(0,2)} \rightarrow T^{(0,1)} T^{A}$ is a free finitely generated module over $C^{\infty}\left(S^{A}\right)$, where $S^{A}$ is a finite dimensional vector space depending canonically on $A$.

Theorem 0.2. Let $A$ be a Weil algebra with $p$ variables. For $n$-manifolds $(n \geq$ $p+3)$, the space of all natural operators $T^{(0,2)} \rightarrow T^{(0,2)} T^{A}$ is a free finitely generated module over $C^{\infty}\left(S^{A}\right)$, where $S^{A}$ is a finite dimensional vector space depending canonically on $A$ (the same as in Theorem 0.1 ).

Received March 25, 1994. 
In the proofs of these theorems we construct explicitly the bases of these $C^{\infty}\left(S^{A}\right)$-modules. The space $S^{A}$ is defined in Section 1 .

Problems of finding all some type natural operators has been studied by many authors, cf. [1], [2], [3], [5]-[14]. Classifications of natural operators $T^{(p, q)} \rightarrow$ $T^{(p, q)} T^{A}$ are useful in the cases: (a) $(p, q)=(0,1)$ because of almost contact structures; $(b)(p, q)=(0,2)$ because of Riemannian and almost symplectic structures; (c) $(p, q)=(1,1)$ because of almost tangent and almost complex structures; (d) $(p, q)=(0,0)$; (e) $(p, q)=(1,0)$ because of vector fields. A classification in the case (e) is given by I. Kolár [5]. Classifications in cases (d) and (e) are presented in [10] and [13] respectively. Hence this paper is a continuation of [5], [10], [13]. A classification in the case (c) is unknown. A classification of all natural operators $T^{(p, q)} \rightarrow T^{(\bar{p}, \bar{q})} T^{A}$ for arbitrary $p, q, \bar{p}, \bar{q}$ is unknown even in the case $T^{A} M=M$.

It will be interesting to obtain classifications of natural operators lifting some other geometrical objects (for example, connections) to the bundles of $A$-velocities. In [14] we have solved the last problem in the case of foliations.

All manifolds and maps in this paper are assumed to be smooth, i.e. infinitely differentiable. Manifolds are assumed to be finite dimensional and without boundaries.

\section{Main examples}

Let $C_{0}^{\infty}\left(\mathbf{R}^{p}\right)$ be the algebra of all germs at 0 of maps $\mathbf{R}^{p} \rightarrow \mathbf{R}$. Let $\underline{A} \varsubsetneqq$ $C_{0}^{\infty}\left(\mathbf{R}^{p}\right)$ be a finite codimensional ideal (with respect to $\mathbf{R}$ ). The factor algebra $A=C_{0}^{\infty}\left(\mathbf{R}^{p}\right) / \underline{A}$ is called a Weil algebra with $p$ variables.

Let $M$ be a manifold. Two maps $g, h: \mathbf{R}^{p} \rightarrow M, g(0)=h(0)=x$, are called to be $A$-equivalent if $\operatorname{germ}_{0}(\varphi \circ g-\varphi \circ h) \in \underline{A}$ for every $\varphi: M \rightarrow \mathbf{R}$. Such an equivalence class is denoted by $j^{A} g$ and called an $A$-velocity on $M$. The set of all $A$-velocities on $M$ is denoted by $T^{A} M$. Then $T^{A} M$ is a fibre bundle over $M$ with projection $j^{A} g \rightarrow g(0)$. Every chart $(U, \varphi)$ on $M$ determines a chart $\tilde{\varphi}$ on $T^{A} M$ over $U$ given by $\tilde{\varphi}\left(j^{A} g\right)=\left(j^{A}\left(\varphi^{1} \circ g\right), \ldots, j^{A}\left(\varphi^{n} \circ g\right)\right) \in A \times \cdots \times A=\mathbf{R}^{n(\operatorname{dim}(A))}$. Every $f: M \rightarrow N$ is extended to $T^{A} f: T^{A} M \rightarrow T^{A} N$ defined by $T^{A} f\left(j^{A} g\right)=j^{A}(f \circ g)$. $T^{A}$ is a product preserving bundle functor from the category of all manifolds and maps into the category of fibered manifolds and fibered maps, cf. [15], [6], [4].

Let $\mathscr{T}^{(0, \alpha)}(M)$ be the $C^{\infty}(M)$-module of all tensor fields of type $(0, \alpha)$ on $M$.

Let $A$ be as above. Let $\mathscr{T}_{0}^{(0, \alpha)}\left(\mathbf{R}^{p}\right)$ be the $C_{0}^{\infty}\left(\mathbf{R}^{p}\right)$-module of all germs at 0 of tensor fields of type $(0, \alpha)$ on $\mathbf{R}^{p}$. Let 


$$
Q^{A}=\mathscr{T}_{0}^{(0,1)}\left(\mathbf{R}^{p}\right) /\left(\underline{A}_{0}^{(0,1)}\left(\mathbf{R}^{p}\right)+C_{0}^{\infty}\left(\mathbf{R}^{p}\right) d \underline{A}\right)
$$

be the factor module, where $A \mathscr{T}_{0}^{(0,1)}\left(\mathbf{R}^{p}\right)$ is the multiplication of $\mathscr{T}_{0}^{(0,1)}\left(\mathbf{R}^{p}\right)$ by $\underline{A}$ and $C_{0}^{\infty}\left(\mathbf{R}^{p}\right) d \underline{A}$ is the submodule of $\mathscr{T}_{0}^{(0,1)}\left(\mathbf{R}^{p}\right)$ spanned by all $d f$ with $f \in \underline{A}$. Let

$$
\begin{aligned}
S^{A}=\mathscr{T}_{0}^{(0,2)}\left(\mathbf{R}^{p}\right) /\left(\underline{A}_{0}^{(0,2)}\left(\mathbf{R}^{p}\right)+(\right. & \left.C_{0}^{\infty}\left(\mathbf{R}^{p}\right) d \underline{A}\right) \otimes \mathscr{T}_{0}^{(0,1)}\left(\mathbf{R}^{p}\right) \\
& \left.+\mathscr{T}_{0}^{(0,1)}\left(\mathbf{R}^{p}\right) \otimes\left(C_{0}^{\infty}\left(\mathbf{R}^{p}\right) d \underline{A}\right)\right)
\end{aligned}
$$

be the factor module, where $\left(C_{0}^{\infty}\left(\mathbf{R}^{p}\right) d \underline{A}\right) \otimes \mathscr{T}_{0}^{(0,1)}\left(\mathbf{R}^{p}\right)$ is the submodule in $\mathscr{T}_{0}^{(0,2)}\left(\mathbf{R}^{p}\right)$ generated by all $\operatorname{germ}_{0}\left(\omega_{1} \otimes \omega_{2}\right)$ with $\operatorname{germ}_{0}\left(\omega_{1}\right) \in C_{0}^{\infty}\left(\mathbf{R}^{p}\right) d \underline{A}$ and $\operatorname{germ}_{0}\left(\omega_{2}\right) \in \mathscr{T}_{0}^{(0,1)}\left(\mathbf{R}^{p}\right)$. Of course, $Q^{A}$ and $S^{A}$ are finite dimensional vector spaces over $\mathbf{R}$. Given $\omega \in \mathscr{T}^{(0,1)}\left(\mathbf{R}^{\not}\right)$ the equivalence class of germ 0 modulo $\underline{A} \mathscr{T}_{0}^{(0,1)}\left(\mathbf{R}^{\not}\right)+C_{0}^{\infty}\left(\mathbf{R}^{p}\right) d \underline{A}$ is denoted by $[\omega]_{A}$. Given $\tau \in \mathscr{T}^{(0,2)}\left(\mathbf{R}^{p}\right)$ the equivalence class of germ $\tau$ modulo $\left.\underline{A}_{0}^{(0,2)}\left(\mathbf{R}^{p}\right)+C_{0}^{\infty}\left(\mathbf{R}^{p}\right) d \underline{A}\right) \otimes \mathscr{T}_{0}^{(0,1)}\left(\mathbf{R}^{p}\right)+$ $\mathscr{T}_{0}^{(0,1)}\left(\mathbf{R}^{p}\right) \otimes\left(C_{0}^{\infty}\left(\mathbf{R}^{p}\right) d \underline{A}\right)$ is denoted by $[[\tau]]_{A}$.

For example in the case $A=C_{0}^{\infty}(\mathbf{R}) /\left\langle t^{r+1}\right\rangle$, where $t$ is the usual coordinate on $\mathbf{R}$ and $\left\langle t^{r+1}\right\rangle$ is the ideal in $C_{0}^{\infty}(\mathbf{R})$ generated by the germ at 0 of $t^{r+1}$, we have $Q^{A}=\left(J^{r-1} T^{(0,1)}\right)_{0} \mathbf{R}=\left\{j_{0}^{r-1} \omega: \omega \in \mathscr{T}^{(0,1)}(\mathbf{R})\right\}$ and $[\omega]_{A}=j_{0}^{r-1} \omega$ for any $\omega$ $\in \mathscr{T}^{(0,1)}(\mathbf{R})$. For, $d t^{r+1}=(r+1) t^{r} d t$, and hence $\left\langle t^{r-1}\right\rangle \mathscr{T}_{0}^{(0,1)}(\mathbf{R})+C_{0}^{\infty}(\mathbf{R}) d$ $\left\langle t^{r+1}\right\rangle=\left\langle t^{r}\right\rangle \mathscr{T}_{0}^{(0,1)}(\mathbf{R})$. Similarly, $S^{A}=\left(J^{r-1} T^{(0,2)}\right)_{0} \mathbf{R}$ and $[[\tau]]_{A}=j_{0}^{r-1} \tau$ for any $\tau \in \mathscr{T}^{(0,2)}(\mathbf{R})$.

In the case $A=C_{0}^{\infty}\left(\mathbf{R}^{2}\right) /\left\langle\left(t^{1}\right)^{2},\left(t^{2}\right)^{2}\right\rangle$, where $t^{1}, t^{2}$ are the usual coordinates on $\mathbf{R}^{2}$ and $\left\langle\left(t^{1}\right)^{2},\left(t^{2}\right)^{2}\right\rangle$ is the ideal in $C_{0}^{\infty}\left(\mathbf{R}^{2}\right)$ generated by the germs at 0 of $\left(t^{1}\right)^{2}$ and $\left(t^{2}\right)^{2}$, we can compute the dimension of $Q^{A}$ as follows. The module $\left\langle\left(t^{1}\right)^{2}\right.$, $\left(t^{2}\right)^{2}>\mathscr{T}_{0}^{(0,1)}\left(\mathbf{R}^{2}\right)$ is generated over $C_{0}^{\infty}\left(\mathbf{R}^{2}\right)$ by the germs at 0 of $\left(t^{1}\right)^{2} d t^{1},\left(t^{1}\right)^{2} d t^{2}$, $\left(t^{2}\right)^{2} d t^{1}$ and $\left(t^{2}\right)^{2} d t^{2}$. Furthermore, $d\left(\left(t^{i}\right)^{2} f\right)=2 f t^{i} d t^{i}+\left(t^{i}\right)^{2} d f$ for any $i=1,2$ and any $f: \mathbf{R}^{2} \rightarrow \mathbf{R}$. Thus the module $\left\langle\left(t^{1}\right)^{2},\left(t^{2}\right)^{2}\right\rangle \mathscr{T}_{0}^{(0,1)}\left(\mathbf{R}^{2}\right)+C_{0}^{\infty}\left(\mathbf{R}^{2}\right) d\left\langle\left(t^{1}\right)^{2}\right.$, $\left.\left(t^{2}\right)^{2}\right\rangle$ is generated over $C_{0}^{\infty}\left(\mathbf{R}^{2}\right)$ by the germs at 0 of $t^{1} d t^{1},\left(t^{1}\right)^{2} d t^{2},\left(t^{2}\right)^{2} d t^{1}$, $t^{2} d t^{2}$. Hence the classes $\left[d t^{1}\right]_{A},\left[d t^{2}\right]_{A},\left[t^{1} d t^{2}\right]_{A}$ and $\left[t^{2} d t^{1}\right]_{A}$ form a basis over $\mathbf{R}$ in $Q^{A}$. Similarly, the classes $\left[\left[d t^{1} \otimes d t^{1}\right]\right]_{A},\left[\left[d t^{1} \otimes d t^{2}\right]\right]_{A},\left[\left[d t^{2} \otimes d t^{1}\right]\right]_{A}$, $\left[\left[d t^{2} \otimes d t^{2}\right]\right]_{A},\left[\left[t^{1} d t^{2} \otimes d t^{2}\right]\right]_{A}$ and $\left[\left[t^{2} d t^{1} \otimes d t^{1}\right]\right]_{A}$ form a basis over $\mathbf{R}$ in $S^{A}$.

Analogously, if $A=C_{0}^{\infty}\left(\mathbf{R}^{p}\right) / m_{0}^{2}$, where $m_{0}$ is the maximal ideal, then the classes $\left[d t^{i}\right]_{A},\left[t^{j} d t^{k}\right]=\frac{1}{2}\left[t^{j} d t^{k}-t^{k} d t^{j}\right]_{A}, i=1, \ldots, p, 1 \leq j<k \leq p$, form a basis of $Q^{A}$ and the classes $\left[\left[d t^{i} \otimes d t^{j}\right]\right]_{A},\left[\left[t^{k} d t^{q} \otimes d t^{s}\right]\right]_{A}, i, j=1, \ldots, p$, $1 \leq k<q<s \leq p$, form a basis of $S^{A}$.

It is difficult to find explicit descriptions for $Q^{A}$ and $S^{A}$ with $p>1$.

Given a tensor field $\tau \in \mathscr{T}^{(0,2)}(M)$ of type $(0,2)$ on $M$ we define $\tau^{s} \in$ 
$\mathscr{T}^{(0,2)}(M)$ by

$$
\left\langle\tau^{s}, u \otimes w\right\rangle=\langle\tau, w \otimes u\rangle, \quad(u, w) \in T M \times_{M} T M .
$$

The linear isomorphism $\mathscr{T}^{(0,2)}\left(\mathbf{R}^{\not p}\right) \ni \tau \rightarrow \tau^{s} \in \mathscr{T}^{(0,2)}\left(\mathbf{R}^{\not}\right)$ induces a linear isomorphism

$$
J_{A}: S^{A} \rightarrow S^{A}, \quad J_{A}\left([[\tau]]_{A}\right)=\left[\left[\tau^{s}\right]\right]_{A} .
$$

Let $\tau \in \mathscr{T}^{(0,2)}(M)$ be a tensor field of type $(0,2)$ on $M$. We present some examples of tensor fields of types $(0,1)$ and $(0,2)$ on $T^{A} M$ induced by $\tau$.

Example 1.1. Let $\phi \in\left(S^{A}\right)^{*}$ be a functional on $S^{A}$. Define

$$
\tau^{[\psi]}: T^{A} M \rightarrow \mathbf{R} \quad \tau^{[\psi]}\left(j^{A} \gamma\right)=\phi\left(\left[\left[\gamma^{*} \tau\right]\right]_{A}\right)
$$

for any $\gamma: \mathbf{R}^{p} \rightarrow M$, where $\gamma^{*} \tau$ is the pull-back of $\tau$ with respect to $\gamma$.

If $\eta: \mathbf{R}^{p} \rightarrow M$ is another map such that $j^{A} \gamma=j^{A} \eta$, then $\left[\left[\gamma^{*} \tau\right]\right]_{A}=\left[\left[\eta^{*} \tau\right]\right]_{A}$. To see this one can assume that $M=\mathbf{R}^{n}$ and $\eta(0)=\gamma(0)=0$. Let $\tau=a_{i j} d x^{i} \otimes$ $d x^{j}, \eta=\left(\eta^{1}, \ldots, \eta^{n}\right)$ and $\gamma=\left(\gamma^{1}, \ldots, \gamma^{n}\right)$. Then $\operatorname{germ}_{0}\left(\eta^{j}-\gamma^{j}\right) \in \underline{A}$ and $\operatorname{germ}_{0}\left(a_{i j}{ }^{\circ} \gamma-a_{i j}{ }^{\circ} \eta\right) \in \underline{A}$ for any $i, j=1, \ldots, n$. Then

$$
\begin{aligned}
\operatorname{germ}_{0}\left(\gamma^{*} \tau-\eta^{*} \tau\right) & =\operatorname{germ}_{0}\left(\left(a_{i j}{ }^{\circ} \gamma-a_{i j}{ }^{\circ} \eta\right) d \gamma^{i} \otimes d \gamma^{j}+a_{\imath \jmath}{ }^{\circ} \eta\left(d\left(\gamma^{i}-\eta^{i}\right)\right) \otimes d \gamma^{j}\right. \\
& \left.+a_{i j}{ }^{\circ} \eta d \eta^{i} \otimes d\left(\gamma^{j}-\eta^{j}\right)\right) \\
& \in \underline{A}_{0}^{(0,2)}\left(\mathbf{R}^{p}\right)+\left(C_{0}^{\infty}\left(\mathbf{R}^{p}\right) d \underline{A}\right) \otimes \mathscr{T}_{0}^{(0,1)}\left(\mathbf{R}^{p}\right) \\
& +\mathscr{T}_{0}^{(0,1)}\left(\mathbf{R}^{p}\right) \otimes\left(C_{0}^{\infty}\left(\mathbf{R}^{p}\right) d \underline{A}\right),
\end{aligned}
$$

as well. Therefore $\tau^{[\psi]}$ is well-defined. One can easily show that this is smooth.

Define $\tau^{\langle\psi\rangle}=d\left(\tau^{[\psi]}\right) \in \mathscr{T}^{(0,1)}\left(T^{A} M\right)$. We observe that $\left(\tau^{s}\right)^{\langle\psi\rangle}=\tau^{\left\langle\mathrm{oJ}_{A}\right\rangle}$, where ()$^{s}$ is described in (1.3) and $J_{A}$ is defined in (1.4).

Example 1.2. Let $\varphi \in\left(Q^{A}\right)^{*}$ be a functional on $Q^{A}$. Define $\tau^{\langle\varphi\rangle_{1}}: T T^{A} M \rightarrow$ $\mathbf{R}$ as follows.

Let $\tilde{A}=C_{0}^{\infty}\left(\mathbf{R}^{p} \times \mathbf{R}\right) /\left\langle\underline{A},(t)^{2}\right\rangle \cong\left(C_{0}^{\infty}\left(\mathbf{R}^{p}\right) / \underline{A}\right) \otimes\left(C_{0}^{\infty}(\mathbf{R}) /\left\langle(t)^{2}\right\rangle\right)$ be the factor algebra, where $t^{1}, \ldots, \overline{t^{p}}, t$ are the usual coordinates on $\mathbf{R}^{p} \times \mathbf{R}$ and $\langle\underline{A}$, $\left.(t)^{2}\right\rangle$ is the ideal in $C_{0}^{\infty}\left(\mathbf{R}^{p} \times \mathbf{R}\right)$ generated by the germ of $(t)^{2}$ at 0 and all $f\left(t^{1}\right.$, $\left.\ldots, t^{p}\right)$ with $f \in \underline{A}$. We identify $T^{\widetilde{A}} M$ with $T T^{A} M$ by a canonical isomorphism, $\left.j^{\tilde{A}} \gamma \rightarrow \frac{d}{d \rho}\right|_{\rho=0}\left(j^{A} \gamma_{\rho}\right)$, where $\gamma: \mathbf{R}^{p} \times \mathbf{R} \rightarrow M, \gamma_{\rho}: \mathbf{R}^{p} \rightarrow M, \gamma_{\rho}=\gamma(\cdot, \rho), \rho \in \mathbf{R}$.

Let $v=j^{\tilde{A}} \gamma \in T T^{A} M$, where $\gamma: \mathbf{R}^{p} \times \mathbf{R} \rightarrow M$. Then $\gamma^{*} \tau \in \mathscr{T}^{(0,2)}\left(\mathbf{R}^{p} \times \mathbf{R}\right)$. Using the contraction $C_{1}^{1}, C_{1}^{1}(\Theta \otimes w)=\Theta(w,$.$) , we have C_{1}^{1}\left(\gamma^{*} \tau \otimes \frac{\partial}{\partial t}\right) \in \mathscr{T}^{(0,1)}$ 
$\left(\mathbf{R}^{p} \times \mathbf{R}\right)$. Then using $i: \mathbf{R}^{p} \rightarrow \mathbf{R}^{p} \times \mathbf{R}, i(x)=(x, 0)$, we get $i^{*}\left(C_{1}^{1}\left(\gamma^{*} \tau \otimes \frac{\partial}{\partial t}\right)\right)$ $\in \mathscr{T}^{(0,1)}\left(\mathbf{R}^{p}\right)$. We put $\tau^{\langle\varphi\rangle_{1}}(v)=\varphi\left(\left[i^{*}\left(C_{1}^{1}\left(\gamma^{*} \tau \otimes \frac{\partial}{\partial t}\right)\right)\right]_{A}\right)$.

If $\eta: \mathbf{R}^{p} \times \mathbf{R} \rightarrow M$ is another map such that $j^{\tilde{A}} \gamma=j^{\tilde{A}} \eta$, then $\left[i^{*}\left(C_{1}^{1}\left(\gamma^{*} \tau \otimes\right.\right.\right.$ $\left.\left.\left.\frac{\partial}{\partial t}\right)\right)\right]_{A}=\left[i^{*}\left(C_{1}^{1}\left(\eta^{*} \tau \otimes \frac{\partial}{\partial t}\right)\right)\right]_{A}$. For, by the reasoning as in Example 1.1 with $\tilde{A}$ instead of $A$ we have

$$
\begin{aligned}
\operatorname{germ}_{0}\left(\gamma^{*} \tau-\eta^{*} \tau\right) & \in \underline{\tilde{A}}_{0}^{(0,2)}\left(\mathbf{R}^{p} \times \mathbf{R}\right)+\left(C_{0}^{\infty}\left(\mathbf{R}^{p} \times \mathbf{R}\right) d \underline{\tilde{A}}\right) \otimes \mathscr{T}_{0}^{(0,1)}\left(\mathbf{R}^{p} \times \mathbf{R}\right) \\
& +\mathscr{T}_{0}^{(0,1)}\left(\mathbf{R}^{p} \times \mathbf{R}\right) \otimes\left(C_{0}^{\infty}\left(\mathbf{R}^{p} \times \mathbf{R}\right) d \underline{\tilde{A}}\right) \\
& \subset \underline{A}_{0}^{(0,2)}\left(\mathbf{R}^{p} \times \mathbf{R}\right)+(t)^{2} \mathscr{T}_{0}^{(0,2)}\left(\mathbf{R}^{p} \times \mathbf{R}\right) \\
& +\left(C_{0}^{\infty}\left(\mathbf{R}^{p} \times \mathbf{R}\right) d \underline{A}\right) \otimes \mathscr{T}_{0}^{(0,1)}\left(\mathbf{R}^{p} \times \mathbf{R}\right)+t d t \otimes \mathscr{T}_{0}^{(0,1)}\left(\mathbf{R}^{p} \times \mathbf{R}\right) \\
& +\mathscr{T}_{0}^{(0,1)}\left(\mathbf{R}^{p} \times \mathbf{R}\right) \otimes\left(C_{0}^{\infty}\left(\mathbf{R}^{p} \times \mathbf{R}\right) d \underline{A}\right)+t \mathscr{T}_{0}^{(0,1)}\left(\mathbf{R}^{p} \times \mathbf{R}\right) \otimes d t,
\end{aligned}
$$

where the germs of $t$ and $d t$ at 0 are also denoted by $t$ and $d t$.

Then

$$
\begin{aligned}
\operatorname{germ}_{0}\left(C_{1}^{1}\left(\left(\gamma^{*} \tau-\eta^{*} \tau\right) \otimes \frac{\partial}{\partial t}\right)\right) & \in \underline{A}_{0}^{(0,1)}\left(\mathbf{R}^{p} \times \mathbf{R}\right)+(t)^{2} \mathscr{T}_{0}^{(0,1)}\left(\mathbf{R}^{p} \times \mathbf{R}\right) \\
& +t \mathscr{T}_{0}^{(0,1)}\left(\mathbf{R}^{p} \times \mathbf{R}\right)+C_{0}^{\infty}\left(\mathbf{R}^{p} \times \mathbf{R}\right) d \underline{A}+t C_{0}^{\infty}\left(\mathbf{R}^{p} \times \mathbf{R}\right) d t
\end{aligned}
$$

because any element from $\underline{A}$ is independent of $t$. Hence

$$
\operatorname{germ}_{0}\left(i^{*}\left(C_{1}^{1}\left(\left(\gamma^{*} \tau-\eta^{*} \tau\right) \otimes \frac{\partial}{\partial t}\right)\right)\right) \in \underline{A}_{0}^{(0,1)}\left(\mathbf{R}^{p}\right)+C_{0}^{\infty}\left(\mathbf{R}^{p}\right) d \underline{A},
$$

as well. Therefore $\tau^{\langle\varphi\rangle_{1}}$ is well-defined. One can easily show that this is smooth. If we consider $\alpha v$ instead of $v$ then $\alpha v=j^{\tilde{A}}\left(\gamma\left(t^{1}, \ldots, t^{p}, \alpha t\right)\right)$, where $v=j^{\tilde{A}} \gamma$. Thus $\alpha \tau^{\langle\varphi\rangle_{1}}(v)=\tau^{\langle\varphi\rangle_{1}}(\alpha v)$. Therefore $\tau^{\langle\varphi\rangle_{1}}$ is linear on each fiber over $T^{A} M$ in view of the homogeneous function theorem. Hence $\tau^{\langle\varphi\rangle_{1}} \in \mathscr{T}^{(0,1)}\left(T^{A} M\right)$.

Let $\tau^{\langle\varphi\rangle_{2}}=\left(\tau^{s}\right)^{\langle\varphi\rangle_{1}}$, where $\tau^{s} \in \mathscr{T}^{(0,2)}(M)$ is given by (1.3).

Example 1.3. Let $\lambda \in A^{*}$ be a functional on $A$. Let $\tau: T M \times{ }_{M} T M \rightarrow \mathbf{R}$ be the tensor field. Let $I: T T^{A} M \rightarrow T^{A} T M$ be the canonical isomorphism given by

$$
I\left(\left.\frac{d}{d t}\right|_{t=0} j^{A} \gamma(\cdot, t)\right)=j^{A}\left(\left.\frac{d}{d t}\right|_{t=0} \gamma(\cdot, t)\right) .
$$

Define $\tau^{\langle\lambda\rangle_{1}}=\lambda \circ T^{A} \tau^{\circ}(I \times I): T T^{A} M \times_{T^{A} M} T T^{A} M \rightarrow \mathbf{R} . \tau^{\langle\lambda\rangle_{1}}$ is a tensor field of 
type $(0,2)$ on $T^{A} M$ called the $\lambda$-lift of $\tau$ to $T^{A} M$, cf. [4].

Let $\tau^{\langle\lambda\rangle_{2}}=\left(\tau^{s}\right)^{\langle\lambda\rangle_{1}}$, where ()$^{s}$ is given by (1.3). We observe that $\tau^{\langle\lambda\rangle_{2}}=$ $\left(\tau^{\langle\lambda\rangle_{1}}\right)^{s}$.

2. Natural operators $T^{(0,2)} \rightarrow T^{(0,1)} T^{A}$ and $T^{(0,2)} \rightarrow T^{(0,2} T^{A}$

It is well-known that the concept of geometrical constructions can be formulated in the form of natural operators, cf. [6].

ExAmple 2.1. Let $n$ be a fixed natural number. Let $A$ be a fixed Weil algebra. Let $\phi \in\left(S^{A}\right)^{*}$. The family $B^{\langle\psi\rangle}$ of functions

$$
B_{M}^{\langle\psi\rangle}: \mathscr{T}^{(0,2)}(M) \rightarrow \mathscr{T}^{(0,1)}\left(T^{A} M\right), \quad B_{M}^{\langle\psi\rangle}(\tau)=\tau^{\langle\psi\rangle},
$$

for any $n$-manifold $M$, where $\tau^{\langle\psi\rangle}$ is described in Example 1.1, is a natural operator $T^{(0,2)} \rightarrow T^{(0,1)} T^{A}$ for $n$-manifolds. For, if $f: M \rightarrow N$ is an embedding of two $n$-manifolds and $\tau \in \mathscr{T}^{(0,2)}(N)$ is a tensor field of type $(0,2)$ on $N$, then $B_{M}^{\langle\psi\rangle}\left(f^{*} \tau\right)=\left(T^{A} f\right)^{*} B_{N}^{\langle\psi\rangle}(\tau)$. Foremore, $B_{M}^{\langle\psi\rangle}$ is regular, i.e. it transforms smoothly parametrized families of tensor fields into smoothly parametrized families.

Example 2.2. Let $n, A$ be as above. Let $\varphi \in\left(Q^{A}\right)^{*}$. For $\alpha=1,2$ the family $B^{\langle\varphi\rangle_{\alpha}}$ of functions

$$
B_{M}^{\langle\varphi\rangle_{\alpha}}: \mathscr{T}^{(0,2)}(M) \rightarrow \mathscr{T}^{(0,1)}\left(T^{A} M\right), \quad B_{M}^{\langle\varphi\rangle_{\alpha}}(\tau)=\tau^{\langle\varphi\rangle_{\alpha}},
$$

for any $n$-manifold $M$, where $\tau^{\langle\varphi\rangle_{\alpha}}$ is described in Example 1.2, is a natural operator $T^{(0,2)} \rightarrow T^{(0,1)} T^{A}$ for $n$-manifolds.

Example 2.3. Let $n, A$ be as above. Let $\lambda \in A^{*}$. For $\alpha=1,2$ the family $C^{\langle\lambda\rangle_{\alpha}}$ of functions

$$
C_{M}^{\langle\lambda\rangle_{\alpha}}: \mathscr{T}^{(0,2)}(M) \rightarrow \mathscr{T}^{(0,2)}\left(T^{A} M\right), \quad C_{M}^{\langle\lambda\rangle_{\alpha}}(\tau)=\tau^{\langle\lambda\rangle_{\alpha}},
$$

for any $n$-manifold $M$, where $\tau^{\langle\lambda\rangle_{\alpha}}$ is described in Example 1.3, is a natural operator $T^{(0,2)} \rightarrow T^{(0,2)} T^{A}$ for $n$-manifolds.

Example 2.4. Let $B, \bar{B}: T^{(0,2)} \rightarrow T^{(0,1)} T^{A}$ be natural operators for $n$-manifolds. Then the families $B \otimes \bar{B}$ and $d B$ of functions

$$
\begin{gathered}
(B \otimes \bar{B})_{M}: \mathscr{T}^{(0,2)}(M) \rightarrow \mathcal{T}^{(0,2)}\left(T^{A} M\right), \quad(B \otimes \bar{B})_{M}(\tau)=\left(B_{M}(\tau)\right) \otimes\left(\bar{B}_{M}(\tau)\right), \\
(d B)_{M}: \mathscr{T}^{(0,2)}(M) \rightarrow \mathcal{T}^{(0,2)}\left(T^{A} M\right), \quad(d B)_{M}(\tau)=d\left(B_{M}(\tau)\right),
\end{gathered}
$$


for any $n$-manifold $M$, are natural operators $T^{(0,2)} \rightarrow T^{(0,2)} T^{A}$ for $n$-manifolds.

\section{The main result}

Let $n$ be a fixed natural number and $A=C_{0}^{\infty}\left(\mathbf{R}^{p}\right) / \underline{A}$ be a fixed Weil algebra. The set of all natural operators $T^{(0,2)} \rightarrow T^{(0,1)} T^{A}$ (or $T^{(0,2)} \rightarrow T^{(0,2)} T^{A}$ ) for $n$-manifolds is a $C^{\infty}\left(S^{A}\right)$-module, where $S^{A}$ is defined in (1.2). Actually, for any $B, C: T^{(0,2)} \rightarrow T^{(0,1)} T^{A}$ (or $\left.B, C: T^{(0,2)} \rightarrow T^{(0,2)} T^{A}\right)$ and $f, g \in C^{\infty}\left(S^{A}\right)$ the natural operator $f B+g C: T^{(0,2)} \rightarrow T^{(0,1)} T^{A}$ (or $f B+g C: T^{(0,2)} \rightarrow T^{(0,2)} T^{A}$ ) is defined by

$$
\begin{aligned}
& \left((f B+g C)_{M}(\tau)\right)\left(j^{A} \gamma\right) \\
& \quad:=f\left(\left[\left[\gamma^{*} \tau\right]\right]_{A}\right)\left(\left(B_{M}(\tau)\right)\left(j^{A} \gamma\right)\right)+g\left(\left[\left[\gamma^{*} \tau\right]\right]_{A}\right)\left(\left(C_{M}(\tau)\right)\left(j^{A} \gamma\right)\right)
\end{aligned}
$$

for any $n$-manifold $M, \tau \in \mathscr{T}^{(0,2)}(M)$ and $\gamma: \mathbf{R}^{p} \rightarrow M$. We showed in Example 1.1 that $\left[\left[\gamma^{*} \tau\right]\right]_{A}$ depends on $j^{A} \gamma$.

In particular, the set of all natural operators $T^{(0,2)} \rightarrow T^{(0,1)} T^{A}$ (or the set of all natural operators $T^{(0,2)} \rightarrow T^{(0,2)} T^{A}$ ) for $n$-manifolds is a vector space over $\mathbf{R}$, provided all real numbers are considered as constant functions on $S^{A}$. The set of all linear natural operators $T^{(0,2)} \rightarrow T^{(0,1)} T^{A}$ (or the set of all linear natural operators $T^{(0,2)} \rightarrow T^{(0,2)} T^{A}$ ) for $n$-manifolds is a vector subspace in this vector space.

The main result of this paper is formulated in the following two theorems corresponding to Theorems 0.1 and 0.2 .

THEOREM 3.1. Let $n$ be a fixed natural number and $A=C_{0}^{\infty}\left(\mathbf{R}^{p}\right) / \underline{A}$ be a fixed Weil algebra. Let $\varphi_{1}, \ldots, \varphi_{s}$ be a basis of the vector space $\left(Q^{A}\right)^{*}$ and $\phi_{1}, \ldots, \phi_{l}$ be a basis of the vector space $\left(S^{A}\right)^{*}$. If $n \geq p+2$, then the natural operators

$$
B^{\left\langle\varphi_{\mu}\right\rangle_{\alpha}}, B^{\left\langle\psi_{\rho}\right\rangle}, \quad \mu=1, \ldots, s, \alpha=1,2, \rho=1, \ldots, l
$$

(described in Examples 2.1 and 2.2) form a basis of the $C^{\infty}\left(S^{A}\right)$-module (described above) of all natural operators $T^{(0,2)} \rightarrow T^{(0,1)} T^{A}$ for $n$-manifolds.

In particular, every natural operator $T^{(0,2)} \rightarrow T^{(0,1)} T^{A}$ for $n$-manifolds is of finite order, provided $n \geq p+2$.

THEOREM 3.2. Let $n$ be a fixed natural number and $A=C_{0}^{\infty}\left(\mathbf{R}^{p}\right) / A$ be a fixed Weil algebra. Let $\varphi_{1}, \ldots, \varphi_{s}$ be a basis of the vector space $\left(Q^{A}\right)^{*}, \phi_{1}, \ldots, \phi_{l}$ be a basis of the vector space $\left(S^{A}\right)^{*}$ and $\lambda_{1}, \ldots, \lambda_{k}$ be a basis of the vector space $A^{*}$. If $n \geq p+3$, then the natural operators 


$$
\begin{aligned}
& C^{\left\langle\lambda_{\sigma}\right\rangle_{\alpha}}, B^{\left\langle\varphi_{\mu}\right\rangle_{\alpha}} \otimes B^{\left\langle\varphi_{\nu}\right\rangle_{\beta}}, B^{\left\langle\varphi_{\mu}\right\rangle_{\alpha}} \otimes B^{\left\langle\phi_{\rho}\right\rangle}, \\
& B^{\left\langle\psi_{\rho}\right\rangle} \otimes B^{\left\langle\varphi_{\mu}\right\rangle_{\alpha}}, B^{\left\langle\psi_{\rho}\right\rangle} \otimes B^{\left\langle\psi_{\pi}\right\rangle}, d B^{\left\langle\varphi_{\mu}\right\rangle_{\alpha}},
\end{aligned}
$$

$\sigma=1, \ldots, k, \alpha, \beta=1,2, \rho, \pi=1, \ldots, l, \mu, \nu=1, \ldots, s$ (described in Examples 2.1-2.4) form a basis of the $C^{\infty}\left(S^{A}\right)$-module (described above) of all natural operators $T^{(0,2)} \rightarrow T^{(0,2)} T^{A}$ for $n$-manifolds.

In particular, every natural operator $T^{(0,2)} \rightarrow T^{(0,2)} T^{A}$ for $n$-manifolds is of finite order, provided $n \geq p+3$.

In Section 1 we computed the dimensions of $Q^{A}$ and $S^{A}$ for some $A$. If $A=$ $C_{0}^{\infty}(\mathbf{R}) /\left\langle t^{r+1}\right\rangle$, then $T^{A} M=T^{r} M=\left\{j_{0}^{r} \gamma: \gamma: \mathbf{R} \rightarrow M\right\}, Q^{A} \cong \mathbf{R}^{r}, S^{A} \cong \mathbf{R}^{r}$, $A \cong \mathbf{R}^{r+1}$. If $A=C_{0}^{\infty}\left(\mathbf{R}^{2}\right) /\left\langle\left(t^{1}\right)^{2},\left(t^{2}\right)^{2}\right\rangle$, then $T^{A} M \cong T T M$ by $j^{A} \gamma \rightarrow$ $\left.\frac{d}{d t^{1}}\right|_{t^{1}=0}\left(\left.\frac{d}{d t^{2}}\right|_{t^{2}=0} \gamma\left(t^{1}, t^{2}\right)\right), Q^{A} \cong \mathbf{R}^{4}, S^{A} \cong \mathbf{R}^{6}, A \cong \mathbf{R}^{4}$. If $A=C_{0}^{\infty}\left(\mathbf{R}^{p}\right) / m_{0}^{2}$, then $T^{A} M=T_{1}^{p} M=\left\{j_{0}^{1} \gamma: \gamma: \mathbf{R}^{p} \rightarrow M\right\}, Q^{A} \cong \mathbf{R}^{p+\left(\frac{p}{2}\right)}, S^{\mathrm{A}} \cong \mathbf{R}^{p^{2}+\left(\begin{array}{l}p \\ 3\end{array}\right.}, A \cong \mathbf{R}^{p+1}$. Hence we have the following Corollary of Theorems 3.1 and 3.2.

COROLlARY 3.1. (a) Let $n \geq 3$ and $r \geq 0$. The module of all natural operators $T^{(0,2)} \rightarrow T^{(0,1)} T^{r}$ for $n$-manifolds is isomorphic to $\left(C^{\infty}\left(\mathbf{R}^{r}\right)\right)^{3 r}$.

(b) Let $n \geq 4$ and $r \geq 0$. The module of all natural operators $T^{(0,2)} \rightarrow T^{(0,2)} T^{r}$ for $n$-manifolds is isomorphic to $\left(C^{\infty}\left(\mathbf{R}^{r}\right)\right)^{9 r^{2}+4 r+2}$.

(c) Let $n \geq 4$. The module of all natural operators $T^{(0,2)} \rightarrow T^{(0,1)}(T T)$ for $n$-manifolds is isomorphic to $\left(C^{\infty}\left(\mathbf{R}^{6}\right)\right)^{14}$.

(d) Let $n \geq 5$. The module of all natural operators $T^{(0,2)} \rightarrow T^{(0,2)}(T T)$ for $n$-manifolds is isomorphic to $\left(C^{\infty}\left(\mathbf{R}^{6}\right)\right)^{212}$.

(e) Let $n \geq p+2$. The module of all natural operators $T^{(0,2)} \rightarrow T^{(0,1)} T_{1}^{p}$ for $n$-manifolds is isomorphic to $\left(C^{\infty}\left(\mathbf{R}^{p^{2}+\left(\frac{p}{3}\right)}\right)\right)^{2\left(p^{2}+\left(\frac{p}{2}\right)\right)+p^{2}+\left(\frac{p}{3}\right)}$.

(f) Let $n \geq p+3$. The module of all natural operators $T^{(0,2)} \rightarrow T^{(0,2)} T_{1}^{p}$ for $n$-manifolds is isomorphic to $\left(C^{\infty}\left(\mathbf{R}^{p^{2}+\left(\frac{p}{3}\right)}\right)\right)^{\left(2\left(p+\left(\frac{p}{2}\right)\right)+p^{2}+\left(\frac{p}{3}\right)\right)^{2}+2\left(p+1+p+\left(\frac{p}{2}\right)\right)}$.

Let us remark that $B^{\langle\varphi\rangle_{\alpha}}, B^{\langle\psi\rangle}, C^{\langle\lambda\rangle_{\alpha}}, d B^{\langle\varphi\rangle_{\alpha}}$ are linear natural operators. Hence from Theorems 3.1 and 3.2 it follows the following fact.

COROLlary 3.2. Let $n$ be a fixed natural number and $A=C_{0}^{\infty}\left(\mathbf{R}^{p}\right) / \underline{A}$ be a fixed Weil algebra. Let $\varphi_{1}, \ldots, \varphi_{s}$ be a basis of the vector space $\left(Q^{A}\right)^{*}, \phi_{1}, \ldots, \phi_{l}$ be the basis of the vector space $\left(S^{A}\right)^{*}$ and $\lambda_{1}, \ldots, \lambda_{k}$ be a basis of the vector space $A^{*}$

(a) If $n \geq p+2$, then the linear natural operators $B^{\left\langle\varphi_{\mu}\right\rangle_{\alpha}}, B^{\left\langle\psi_{\rho}\right\rangle}, \mu=1, \ldots, s$, $\alpha=1,2, \rho=1, \ldots, l$, form a basis (over $\mathbf{R}$ ) of the vector space of all linear natural 
operators $T^{(0,2)} \rightarrow T^{(0,1)} T^{A}$ for $n$-manifolds.

(b) If $n \geq p+3$, then the linear natural operators $C^{\left\langle\lambda_{\sigma}\right\rangle_{\alpha}}, d B^{\left\langle\varphi_{\mu}\right\rangle_{\alpha}}, \mu=1, \ldots, s$, $\alpha=1,2, \rho=1, \ldots, k$, form a basis (over $\mathbf{R}$ ) of the vector space of all linear natural operators $T^{(0,2)} \rightarrow T^{(0,2)} T^{A}$ for $n$-manifolds.

Proof of Corollary 3.2. Let $B: T^{(0,2)} \rightarrow T^{(0,2)} T^{A}$ (or $B: T^{(0,2)} \rightarrow T^{(0,2)} T^{A}$ ) be a linear natural operator. Then $B$ is a linear combination of the natural operators presented in Theorem 3.1 (or in Theorem 3.2) with the uniquely determined coefficients from $C^{\infty}\left(S^{A}\right)$. Since $B$ is linear, the coefficients are constant (or the coefficients corresponding to $C^{\left\langle\lambda_{\sigma}\right\rangle_{\alpha}}, d B^{\left\langle\varphi_{\mu}\right\rangle_{\alpha}}$ are constant and the other ones are zero). For, since $B$ is linear, the coefficients are homogeneous of some weights $(0$ or -1 ), and next we use the homogeneous function theorem, cf. [6].

As a consequence of Corollary 3.2 and Theorems 3.1 and 3.2 we obtain

Corollary 3.3. Let $n$ be a fixed natural number and $A=C_{0}^{\infty}\left(\mathbf{R}^{p}\right) / \underline{A}$ be a fixed Weil algebra. Let $B_{1}, \ldots, B_{S}$ be a basis (over $\mathbf{R}$ ) of the vector space of all linear natural operators $T^{(0,2)} \rightarrow T^{(0,1)} T^{A}$ for $n$-manifolds. Let $C_{1}, \ldots, C_{Q}$ be a basis (over $\left.\mathbf{R}\right)$ of the vector space of all linear natural operators $T^{(0,2)} \rightarrow T^{(0,2)} T^{A}$ for $n$-manifolds.

(a) If $n \geq p+2$, then the natural operators $B_{1}, \ldots, B_{S}$ form a basis of the $C^{\infty}\left(S^{A}\right)$-module of all natural operators $T^{(0,2)} \rightarrow T^{(0,1)} T^{A}$ for $n$-manifolds.

(b) If $n \geq p+3$, then the natural operators $C_{\rho}, B_{\nu} \otimes B_{\mu}, \rho=1, \ldots, Q, \nu, \mu$ $=1, \ldots, S$, form a basis of the $C^{\infty}\left(S^{A}\right)$-module of all natural operators $T^{(0,2)} \rightarrow$ $T^{(0,2)} T^{A}$ for $n$-manifolds.

The proofs of Theorems 3.1 and 3.2 are given in Sections 4 and 5.

The purpose of the rest of this section is to explain Theorems 3.1 and 3.2 in the case $T^{r} M$. We do not use this explanation in the proofs of Theorems 3.1 and 3.2 .

Example 3.1. Let $\nu=0,1, \ldots, r$. Given $f: M \rightarrow \mathbf{R}$ one can define $f^{(\nu)}$ : $T^{r} M \rightarrow \mathbf{R}$ by $f^{(\nu)}\left(j_{0}^{r} \gamma\right)=\frac{1}{\nu !} D^{\nu}(f \circ \gamma)(0)$. Given a vector field $X$ on $M$ there exists one and only one vector field $X^{(\nu)}$ on $T^{r} M$ such that $X^{(\nu)} f^{(\mu)}=(X f)^{(\nu+\mu-r)}$ for any $f: M \rightarrow \mathbf{R}$ and $\mu=0, \ldots, r\left(f^{(\mu)}:=0\right.$, if $\mu<0$ or $\left.\mu>r\right)$. Given a 1 -form $\omega$ on $M$ we denote the $\nu$-lift of $\omega$ to $T^{r} M$ in the sense of A. Morimoto [16] by $\omega^{(\nu)}$. Then $\left\langle\omega^{(\nu)}, X^{(\mu)}\right\rangle=\langle\omega, X\rangle^{(\nu+\mu-r)}$ for any vector field $X$ on $M$ and $\mu=$ 


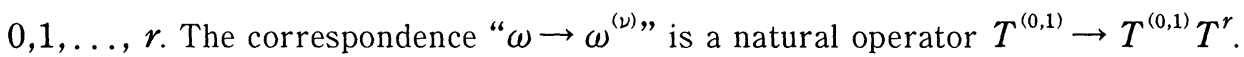

For any tensor field $\tau$ of type $(0,2)$ on $M$ we denote the $\nu$-lift of $\tau$ to $T^{r} M$ in the sense of [16] by $\tau^{(\nu)}$. Then $\left\langle\tau^{(\nu)}, X^{(\mu)} \otimes Y^{(\rho)}\right\rangle=\langle\tau, X \otimes Y\rangle^{(\nu+\mu+\rho-2 r)}$ for any vector fields $X, Y$ on $M$ and any $\mu, \rho=0, \ldots, r$. The correspondence $C^{(\nu)}: T^{(0,2)}$ $\rightarrow T^{(0,2)} T^{r}, \tau \rightarrow \tau^{(\nu)}$, is a linear natural operator. Let $D^{(\nu)}: T^{(0,2)} \rightarrow T^{(0,2)} T^{r}$ be a linear natural operator given by $D_{M}^{(\nu)}(\tau)=C_{M}^{(\nu)}\left(\tau^{s}\right)$, where ()$^{s}$ is defined by (1.3).

ExAmple 3.2. Let $\sigma=0, \ldots, r-1$ and $\alpha=0,1,2$. Let $\pi_{M}: T M \rightarrow M$ denotes the tangent bundle projection. Let $\tau \in \mathscr{T}^{(0,2)}(M)$. Define $\tau^{((\alpha))}$ $\in \mathscr{T}^{(0,1)}(T M)$ by: $\tau^{((0))}=d \tilde{\tau}$, where $\tilde{\tau}: T M \rightarrow \mathbf{R}, \tilde{\tau}(v)=\langle\tau, v \otimes v\rangle ;\left\langle\tau^{((1))}\right.$, $u\rangle=\left\langle\tau, \quad\left(\pi_{T M}(u)\right) \otimes\left(T \pi_{M}(u)\right)\right\rangle, \quad u \in T T M ;\left\langle\tau^{((2))}, \quad u\right\rangle=\left\langle\tau, \quad\left(T \pi_{M}(u)\right) \otimes\right.$ $\left.\left(\pi_{T M}(u)\right)\right\rangle, u \in T T M$. We put $\tau^{(\sigma, \alpha)}=j_{M}^{*}\left(\left(\tau^{((\alpha))}\right)^{(\sigma)}\right) \in \mathscr{T}^{(0,1)}\left(T^{r} M\right)$, where $j_{M}$ : $T^{r} M \rightarrow T^{r-1} T M$ is the canonical embedding given by $j_{0}^{r} \gamma \rightarrow j_{0}^{(r-1)}\left(\left.t \rightarrow \frac{d}{d \tau}\right|_{\tau=0} \gamma(t+\right.$ $\tau))$ and $\left(\tau^{((\alpha))}\right)^{(\sigma)}$ denotes the $\sigma$-lift of $\tau^{((\alpha))} \in \mathscr{T}^{(0,1)}(T M)$ to $T^{r-1} T M$ described in Example 3.1. It is clear that for $\alpha=0,1,2$ and $\sigma=0, \ldots, r-1$ the correspondence $B^{(\sigma, \alpha)}: T^{(0,2)} \rightarrow T^{(0,1)} T^{r}, \tau \rightarrow \tau^{(\sigma, \alpha)}$ is a linear natural operator. By Example 2.4 we have a linear natural operator $d B^{(\sigma, \alpha)}: T^{(0,2)} \rightarrow T^{(0,2)} T^{r}$.

LeMma 3.1. (a) If $n \geq 2$, then the linear natural operators $B^{(\sigma, \alpha)}, \sigma=0, \ldots$, $r-1, \alpha=0,1,2$, are linearly independent in the vector space of all linear natural operators $T^{(0,2)} \rightarrow T^{(0,1)} T^{r}$ for $n$-manifolds.

(b) If $n \geq 3$, then the linear natural operators $C^{(\nu)}, D^{(\nu)}, d B^{(\sigma, \alpha)}, \nu=0, \ldots, r$, $\sigma=0, \ldots, r-1, \alpha=1,2$, are linearly independent in the vector space of all linear natural operators $T^{(0,2)} \rightarrow T^{(0,2)} T^{r}$ for $n$-manifolds.

Proof of Lemma 3.1. Let $x^{1}, \ldots, x^{n}$ be the usual coordinates on $\mathbf{R}^{n}$ and let $\partial_{n}=\frac{\partial}{\partial x^{n}}$. Denote the induced coordinates on $T \mathbf{R}^{n}=\mathbf{R}^{n} \times \mathbf{R}^{n}$ by $x^{1}, \ldots, x^{n}, v^{1}$ $=d x^{1}, \ldots, v^{n}=d x^{n}$. Let $e=j_{0}^{r}(t, 0, \ldots, 0) \in T_{0}^{r} \mathbf{R}^{n}$, where $t$ is the usual coordinate on $\mathbf{R}$. We use the notations of Examples 3.1 and 3.2. Of course, $j_{\mathbf{R}^{n}}(e)=$ $j_{0}^{r-1}(t, 0, \ldots, 0,1,0, \ldots, 0), 1$ in $n+1$-position.

ad(a) Consider $\tau=x^{n} g\left(x^{1}\right) d x^{1} \otimes d x^{1}+f\left(x^{1}\right) d x^{1} \otimes d x^{n}+h\left(x^{1}\right) d x^{n} \otimes d x^{1}$ $\in \mathscr{T}^{(0,2)}\left(\mathbf{R}^{n}\right)$, where $f, g, h: \mathbf{R} \rightarrow \mathbf{R}$. In the induced coordinates, $\tilde{\tau}=$ $x^{n} g\left(x^{1}\right)\left(v^{1}\right)^{2}+f\left(x^{1}\right) v^{1} v^{n}+h\left(x^{1}\right) v^{n} v^{1}$ and $\partial_{n}^{c}=\frac{\partial}{\partial x^{n}}$, where $X^{C}$ is the complete lift of $X$ to $T M$ (defined by the lift of flows). Hence $\left\langle\tau^{((0))}, \partial_{n}^{C}\right\rangle=\partial_{n}^{C} \tilde{\tau}=$ 
$g\left(x^{1}\right)\left(v^{1}\right)^{2}$. Therefore

$$
\begin{aligned}
\left\langle B_{\mathbf{R}^{n}}^{(\sigma, 0)}(\tau), \partial_{n}^{(r)}(e)\right\rangle & =\left\langle\left(\tau^{((0))}\right)^{(\sigma)},\left(\partial_{n}^{c}\right)^{(r-1)}\right\rangle\left(j_{\mathbf{R}^{n}}(e)\right) \\
& =\left\langle\tau^{((0))}, \partial_{n}^{c}\right\rangle^{(\sigma)}\left(j_{\mathbf{R}^{n}}(e)\right)=\frac{1}{\sigma !} \frac{d^{\sigma} g}{d t^{\sigma}}(0) .
\end{aligned}
$$

By the definitions of $\tau^{((\alpha))}$ we have $\left\langle\tau^{((1))}, \partial_{n}^{C}\right\rangle=f\left(x^{1}\right) v^{1}$ and $\left\langle\tau^{((2))}, \partial_{n}^{C}\right\rangle=$ $h\left(x^{1}\right) v^{1}$. Then

$$
\left\langle B_{\mathbf{R}^{n}}^{(\sigma, 1)}(\tau), \partial_{n}^{(r)}(e)\right\rangle=\frac{1}{\sigma !} \frac{d^{\sigma} f}{d t^{\sigma}}(0),\left\langle B_{\mathbf{R}^{n}}^{(\sigma, 2)}(\tau), \partial_{n}^{(r)}(e)\right\rangle=\frac{1}{\sigma !} \frac{d^{\sigma} h}{d t^{\sigma}}(0) .
$$

The case (a) is a simple consequence of formulas (3.1) and (3.2).

ad(b) Consider

$$
\begin{aligned}
\tau & =f\left(x^{1}\right) d x^{n-1} \otimes d x^{n}+g\left(x^{1}\right) d x^{n} \otimes d x^{n-1} \\
& +h\left(x^{1}\right)\left(x^{n} d x^{1} \otimes d x^{n-1}-x^{n-1} d x^{1} \otimes d x^{n}\right) \\
& +k\left(x^{1}\right)\left(x^{n} d x^{n-1} \otimes d x^{1}-x^{n-1} d x^{n} \otimes d x^{1}\right) .
\end{aligned}
$$

Then $\left\langle\tau^{((1))}, \partial_{n}^{c}\right\rangle=f\left(x^{1}\right) v^{n-1}-h\left(x^{1}\right) x^{n-1} v^{1},\left\langle\tau^{((1))}, \partial_{n-1}^{c}\right\rangle=g\left(x^{1}\right) v^{n}+h\left(x^{1}\right) x^{n} v^{1}$. Since $\partial_{n}^{(r)}$ is equal to the complete lift of $\partial_{n}$ to $T^{r} \mathbf{R}^{n}$ and $j_{M}: T^{r} M \rightarrow T^{r-1} T M$ is a natural transformation, then $T j_{\mathbf{R}^{n}} \circ \partial_{n}^{(r)}=\left(\partial_{n}^{C}\right)^{(r-1)} \circ j_{\mathbf{R}^{n}}$. Therefore

$$
\begin{aligned}
& \left\langle\left(d B^{(\sigma, 1)}\right)_{\mathbf{R}^{n}}(\tau),\left(\partial_{n-1}^{(r)}(e)\right) \otimes\left(\partial_{n}^{(r)}(e)\right)\right\rangle \\
& =\left(\partial_{n-1}^{(r)}\left(\left\langle\tau^{(\sigma, 1)}, \partial_{n}^{(r)}\right\rangle\right)-\partial_{n}^{(r)}\left(\left\langle\tau^{(\sigma, 1)}, \partial_{n-1}^{(r)}\right\rangle\right)\right)(e) \\
& =\left(\left(\partial_{n-1}^{C}\right)^{(r-1)}\left(\left\langle\tau^{((1))}, \partial_{n}^{C}\right\rangle\right)^{(\sigma)}-\left(\partial_{n}^{C}\right)^{(r-1)}\left(\left\langle\tau^{((1))}, \partial_{n-1}^{C}\right\rangle\right)^{(\sigma)}\right)\left(j_{\mathbf{R}^{n}}(e)\right) \\
& =\left(\left(\partial_{n-1}^{C}\left(\left\langle\tau^{((1))}, \partial_{n}^{C}\right\rangle\right)\right)^{(\sigma)}-\left(\partial_{n}^{C}\left(\left\langle\tau^{((1))}, \partial_{n-1}^{C}\right\rangle\right)\right)^{(\sigma)}\right)\left(j_{\mathbf{R}^{n}}(e)\right) \\
& =-2\left(h\left(x^{1}\right) v^{1}\right)^{(\sigma)}\left(j_{\mathbf{R}^{n}}(e)\right)=-\frac{2}{\sigma !} \frac{d^{\sigma} h}{d t^{\sigma}}(0) .
\end{aligned}
$$

Similarly,

$$
\left\langle\left(d B^{(\sigma, 2)}\right)_{\mathbf{R}^{n}}(\tau),\left(\partial_{n-1}^{(r)}(e)\right) \otimes\left(\partial_{n}^{(r)}(e)\right)\right\rangle=-\frac{2}{\sigma !} \frac{d^{\sigma} k}{d t^{\sigma}}(0) .
$$

Foremore

$$
\begin{gathered}
\left\langle C_{\mathbf{R}^{n}}^{(\nu)}(\tau),\left(\partial_{n-1}^{(r)}(e)\right) \otimes\left(\partial_{n}^{(r)}(e)\right)\right\rangle=\left(\left\langle\tau, \partial_{n-1} \otimes \partial_{n}\right\rangle\right)^{(\nu)}(e)=\left(f\left(x^{1}\right)\right)^{(\nu)}(e)=\frac{1}{\nu !} \frac{d^{\nu} f}{d t^{\nu}}(0) \\
\left\langle D_{\mathbf{R}^{n}}^{(\nu)}(\tau),\left(\partial_{n-1}^{(r)}(e)\right) \otimes\left(\partial_{n}^{(r)}(e)\right)\right\rangle=\frac{1}{\nu !} \frac{d^{\nu} g}{d t^{\nu}}(0)
\end{gathered}
$$

The case (b) is proved. 
Using Corollary 3.2, Lemma 3.1 and the dimension argument we deduce that the operators of Lemma 3.1(a) and Lemma 3.1(b) form the bases of the vector spaces of all linear natural operators $T^{(0,2)} \rightarrow T^{(0,1)} T^{\mathrm{r}}$ and $T^{(0,2)} \rightarrow T^{(0,2)} T^{r}$ respectively. Hence we have the following consequence of Corollary 3.3 .

Corollary 3.4. (a) Let $n \geq 3$ and $r \geq 0$ be fixed integers. Let $B: T^{(0,2)} \rightarrow$ $T^{(0,1)} T^{r}$ be a natural operator for $n$-manifolds. Then there exist the uniquely determined smooth maps $f_{\sigma \alpha}:\left(J^{r-1} T^{(0,2)}{ }_{0} \mathbf{R} \cong \mathbf{R}^{r} \rightarrow \mathbf{R}, \sigma=0, \ldots, r-1, \alpha=0,1,2\right.$ such that

$$
B_{M}(\tau)\left(j_{0}^{r} \gamma\right)=\sum_{\alpha=0}^{2} \sum_{\sigma=0}^{r-1} f_{\sigma \alpha}\left(j_{0}^{r-1}\left(\gamma^{*} \tau\right)\right) \tau^{(\sigma, \alpha)}\left(j_{0}^{r} \gamma\right)
$$

for any $n$-manifold $M$, any $\tau \in \mathscr{T}^{(0,2)}(M)$ and any $\gamma: \mathbf{R} \rightarrow M$, where $\tau^{(\sigma, \alpha)}$ is described in Example 3.2. If $B$ is linear, then the maps $f_{\sigma \alpha}$ are constant.

(b) Let $n \geq 4$ and $r \geq 0$ be fixed integers. Let $B: T^{(0,2)} \rightarrow T^{(0,2)} T^{r}$ be a natural operator for $n$-manifolds. Then there exist the uniquely determined smooth maps $f_{\nu}, g_{\nu}$, $h_{\sigma \alpha}, \quad H_{\sigma \overline{\sigma \beta} \bar{\beta}}:\left(J^{r-1} T^{(0,2)}\right)_{0} \mathbf{R} \cong \mathbf{R}^{r} \rightarrow \mathbf{R}, \quad \sigma, \bar{\sigma}=0, \ldots, r-1, \nu=0, \ldots, r, \alpha=$ $1,2, \beta, \bar{\beta}=0,1,2$ such that

$$
\begin{aligned}
B_{M}(\tau)\left(j_{0}^{r} \gamma\right) & =\sum_{\alpha=1}^{2} \sum_{\sigma=0}^{r-1} h_{\sigma \alpha}\left(j_{0}^{\gamma-1}\left(\gamma^{*} \tau\right)\right) d \tau^{(\sigma, \alpha)}\left(j_{0}^{r} \gamma\right) \\
& +\sum_{\nu=0}^{r} f_{\nu}\left(j_{0}^{\gamma-1}\left(\gamma^{*} \tau\right)\right) \tau^{(\nu)}\left(j_{0}^{r} \gamma\right)+\sum_{\nu=0}^{r} g_{\nu}\left(j_{0}^{r-1}\left(\gamma^{*} \tau\right)\right)\left(\tau^{s}\right)^{(\nu)}\left(j_{0}^{r} \gamma\right) \\
& +\sum_{\beta, \bar{\beta}=0}^{2} \sum_{\sigma, \bar{\sigma}=0}^{r-1} H_{\sigma \bar{\sigma} \beta \bar{\beta}}\left(j_{0}^{\gamma-1}\left(\gamma^{*} \tau\right)\right)\left(\tau^{(\sigma, \beta)} \otimes \tau^{(\bar{\sigma}, \bar{\beta})}\right)\left(j_{0}^{\gamma} \gamma\right)
\end{aligned}
$$

for any $n$-manifold $M$, any $\tau \in \mathscr{T}^{(0,2)}(M)$ and any $\gamma: \mathbf{R} \rightarrow M$, where $\tau^{(\sigma, \beta)}$ is described in Example 3.2 and $\tau^{(\nu)},\left(\tau^{s}\right)^{(\nu)}$ are described in Example 3.1.

Remark. A classification of all first order natural operators $T^{(0,2)} \rightarrow T^{(0,2)} T_{1}^{k}$, where $T_{1}^{k} M=J_{0}^{1}\left(\mathbf{R}^{k}, M\right)$, has been studied by M. Doupovec and J. Kurek [1].

\section{A preparatory proposition}

We shall be proving Theorems 3.1 and 3.2 simultaneously. Parentheses will deal with Theorem 3.2. In the proofs of Theorems 3.1 and 3.2 we shall use some technical facts proved in this section.

From now on for $\alpha=1,2$ the $C^{\infty}\left(S^{A}\right)$-module of all natural operators $T^{(0,2)}$ $\rightarrow T^{(0, \alpha)} T^{A}$ for $n$-manifolds is denoted by $\mathscr{T}(A, n, \alpha)$. 
Let $x^{1}, \ldots, x^{n}$ be the usual coordinates on $\mathbf{R}^{n}, t^{1}, \ldots, t^{p}$ be the usual coordinates on $\mathbf{R}^{p}, n>p$. Let $\partial_{i}=\frac{\partial}{\partial x^{i}}, i=1, \ldots, n$, be the canonical vector fields on
$\mathbf{R}^{n}$. Let

$$
e:=j^{A}\left(t^{1}, \ldots, t^{p}, 0, \ldots, 0\right) \in T_{0}^{A} \mathbf{R}^{n}
$$

Given a natural operator $B \in \mathscr{T}(A, n, 1)$ (or $B \in \mathscr{T}(A, n, 2)$ ) we define $\Phi_{B}$ $: \mathscr{T}^{(0,2)}\left(\mathbf{R}^{n}\right) \rightarrow \mathbf{R}$ by

$$
\begin{gathered}
\Phi_{B}(\tau):=\left\langle\left(B_{\mathbf{R}^{n}}(\tau)\right)(e), T^{A} \partial_{n}(e)\right\rangle \text { (or } \\
\left.\Phi_{B}(\tau):=\left\langle\left(B_{\mathbf{R}^{n}}(\tau)\right)(e),\left(T^{A} \partial_{n-1}(e)\right) \otimes\left(T^{A} \partial_{n}(e)\right)\right\rangle\right),
\end{gathered}
$$

where $T^{A} X$ is the complete lift of a vector field $X$ on $M$ to $T^{A} M$. If $\varphi_{t}$ is the flow of $X$, then $T^{A} \varphi_{t}$ is the flow of $T^{A} X$.

We start with the proof of the following lemma.

Lemma 4.1. Let $B, C \in \mathscr{T}(A, n, 1), n \geq p+1$ (or $B, C \in \mathscr{T}(A, n, 2), n$ $\geq p+2$ ). If $\Phi_{B}=\Phi_{C}$, then $B=C$.

Proof of Lemma 4.1. It is well-known (see Example 1.2) that $T T^{A} M=T^{\widetilde{A}} M$, where $\tilde{A}=A \otimes\left(C_{0}^{\infty}(\mathbf{R}) /\left\langle(t)^{2}\right\rangle\right) \cong C_{0}^{\infty}\left(\mathbf{R}^{p} \times \mathbf{R}\right) /\left\langle\underline{A},(t)^{2}\right\rangle$, where $t^{1}, \ldots, t^{p}, t$ are the usual coordinates on $\mathbf{R}^{p} \times \mathbf{R}$. Similarly, $T T^{A} M \times{ }_{T^{A} M} T T^{A} M=T^{\bar{A}} M$, where $\bar{A}=C_{0}^{\infty}\left(\mathbf{R}^{p} \times \mathbf{R}^{2}\right) /\left\langle\underline{A},(\bar{t})^{2}, \bar{t} t,(t)^{2}\right\rangle$, where $t^{1}, \ldots, t^{p}, t, \bar{t}$ are the usual coordinates on $\mathbf{R}^{p} \times \mathbf{R}^{2}$. (Coordinates $t^{1}, \ldots, t^{p}$ on $\mathbf{R}^{p}$ we identify (in obvious way) with the coordinates $t^{1}, \ldots, t^{p}$ on $\mathbf{R}^{p} \times \mathbf{R}$ or on $\left.\mathbf{R}^{p} \times \mathbf{R}^{2}\right)$. Of course; $T^{A} \partial_{n}(e)=j^{\tilde{A}}\left(t^{1}, \ldots\right.$, $\left.t^{p}, 0, \ldots, 0, t\right)$ (or $\left(T^{A} \partial_{n-1}(e), T^{A} \partial_{n}(e)\right)=j^{\bar{A}}\left(t^{1}, \ldots, t^{p}, 0, \ldots, 0, t, \bar{t}\right)$ ). If $n \geq$ $p+1$ (or $n \geq p+2$ ), then by the rank theorem the orbit of $j^{\tilde{A}}\left(t^{1}, \ldots, t^{p}, 0, \ldots, 0\right.$, $t$ ) (or of $j^{\bar{A}}\left(t^{1}, \ldots, t^{p}, 0, \ldots, 0, t, \bar{t}\right)$ ) with respect to lifted diffeomorphisms is dense in $\left(T T^{A}\right){ }_{0} \mathbf{R}^{n}$ (or in $\left(\left(T T^{A}\right){ }_{0} \mathbf{R}^{n}\right) \times{ }_{T^{A}} \mathbf{R}^{n}\left(\left(T T^{A}\right){ }_{0} \mathbf{R}^{n}\right)$ ). Using the assumption and the invariancy of $B$ and $C$ we deduce that $\left\langle B_{\mathbf{R}^{n}}(\tau), v\right\rangle=\left\langle C_{\mathbf{R}^{n}}(\tau), v\right\rangle$ (or $\left.\left\langle B_{\mathbf{R}^{n}}(\tau), v \otimes w\right\rangle=\left\langle C_{\mathbf{R}^{n}}(\tau), v \otimes w\right\rangle\right)$ for any $\tau \in \mathscr{T}^{(0,2)}\left(\mathbf{R}^{n}\right)$ and any $v$ (or $(v, w)$ ) from some dense subset in $\left(T T^{A}\right)_{0} \mathbf{R}^{n}$ (or in $\left(\left(T T^{A}\right){ }_{0} \mathbf{R}^{n}\right) \times{ }_{T^{A}} \mathbf{R}^{n}\left(\left(T T^{A}\right)_{0} \mathbf{R}^{n}\right)$ ) Then $B_{\mathbf{R}^{n}}(\tau)=C_{\mathbf{R}^{n}}(\tau)$ over 0 for any $\tau \in \mathscr{T}^{(0,2)}\left(\mathbf{R}^{n}\right)$. Using the invariancy of $B$ and $C$ with respect to charts we get that $B_{M}(\tau)=C_{M}(\tau)$ for any $M$ and any $\tau \in \mathscr{T}^{(0,2)}(M)$, i.e. $B=C$.

Lemma 4.2. Let $B, C \in \mathscr{T}(A, n, 1), n \geq p+1$ (or $B, C \in \mathscr{T}(A, n, 2), n$ $\geq p+2)$. If $\Phi_{B}(\tilde{\tau})=\Phi_{C}(\tilde{\tau})$ for any $\tilde{\tau} \in \mathscr{T}^{(0,2)}\left(\mathbf{R}^{n}\right)$ of the form 


$$
\begin{gathered}
\quad \tilde{\tau}=q^{*} \tau_{0}+x^{n} q^{*} \tau_{1}+\left(q^{*} \omega_{0}\right) \otimes d x^{n}+d x^{n} \otimes\left(q^{*} \omega_{1}\right)(\text { or } \\
\tilde{\tau}=q^{*} \tau_{0}+x^{n} q^{*} \tau_{1}+x^{n-1} q^{*} \tau_{2}+x^{n-1} x^{n} q^{*} \tau_{3}+\left(q^{*} \omega_{0}\right) \otimes d x^{n} \\
+d x^{n} \otimes\left(q^{*} \omega_{1}\right)+\left(q^{*} \omega_{2}\right) \otimes d x^{n-1}+d x^{n-1} \otimes\left(q^{*} \omega_{3}\right) \\
+x^{n}\left(q^{*} \omega_{4}\right) \otimes d x^{n-1}+x^{n-1}\left(q^{*} \omega_{5}\right) \otimes d x^{n}+x^{n} d x^{n-1} \otimes\left(q^{*} \omega_{6}\right) \\
+x^{n-1} d x^{n} \otimes\left(q^{*} \omega_{7}\right)+f_{0}\left(x^{1}, \ldots, x^{p}\right) d x^{n-1} \otimes d x^{n} \\
\left.+f_{1}\left(x^{1}, \ldots, x^{p}\right) d x^{n} \otimes d x^{n-1}\right),
\end{gathered}
$$

where $\tau_{0}, \tau_{1} \in \mathscr{T}^{(0,2)}\left(\mathbf{R}^{p}\right), \omega_{0}, \omega_{1} \in \mathscr{T}^{(0,1)}\left(\mathbf{R}^{p}\right)\left(\right.$ or $\tau_{0}, \ldots, \tau_{3} \in \mathscr{T}^{(0,2)}\left(\mathbf{R}^{p}\right), \omega_{0}, \ldots$, $\left.\omega_{7} \in \mathscr{T}^{(0,1)}\left(\mathbf{R}^{p}\right), f_{0}, f_{1}: \mathbf{R}^{p} \rightarrow \mathbf{R}\right)$ and $q: \mathbf{R}^{n}=\mathbf{R}^{p} \times \mathbf{R}^{n-p} \rightarrow \mathbf{R}^{p}$ is the projection, then $B=C$.

Proof of Lemma 4.2. Let

$$
\tau=\sum_{i, j=1}^{n} f_{i j} d x^{i} \otimes d x^{j}
$$

be an arbitrary tensor field of type $(0,2)$ on $\mathbf{R}^{n}$. By Lemma 4.1 it is sufficient to show that $\Phi_{B}(\tau)=\Phi_{C}(\tau)$.

By the corollary of the non-linear Peetre theorem by Slovák, cf. Corollary 19.8 in [6], there is a natural number $s=s(\tau)$ such that $\Phi_{B}(\bar{\tau})=\Phi_{B}(\tau)$ and $\Phi_{C}(\bar{\tau})=\Phi_{C}(\tau)$ for any $\bar{\tau} \in \mathscr{T}^{(0,2)}\left(\mathbf{R}^{n)}\right.$ with $j_{0}^{s} \bar{\tau}=j_{0}^{s} \tau$. Hence we can assume that $f_{i j}$ are polynomials in $x^{1}, \ldots, x^{n}$ of degree $\leq s$. Then we can write

$$
f_{i j}=\sum_{|\alpha| \leq s} a_{i j \alpha} x^{\alpha}, \quad \alpha=\left(\alpha_{1}, \ldots, \alpha_{n}\right) \in(\mathbf{N} \cup\{0\})^{n} .
$$

Let us denote the restriction of $\Phi_{B}$ to the finite dimensional vector space of all tensor fields type $(0,2)$ on $\mathbf{R}^{n}$ of the form (4.4) with $f_{i j}$ of the form (4.5) by $\Phi_{B}^{s}$. Since $B$ is regular, $\Phi_{B}^{s}$ is smooth with respect to the $a_{i j \alpha}$.

For any $a_{p+1}, \ldots, a_{n} \in \mathbf{R}-\{0\}$ the diffeomorphism $\eta=\left(x^{1}, \ldots, x^{p}\right.$, $\left.a_{p+1} x^{p+1}, \ldots, a_{n} x^{n}\right): \mathbf{R}^{n} \rightarrow \mathbf{R}^{n}$ preserves $e$ and sends $\partial_{n}$ into $a_{n} \partial_{n}$ (or $\partial_{n-1}, \partial_{n}$ into $a_{n-1} \partial_{n-1}, a_{n} \partial_{n}$ ). Using the invariancy of $B$ with respect to $\eta$ we deduce that

$$
a_{n} \Phi_{B}^{s}(\tau)=\Phi_{B}^{s}\left(\eta^{*} \tau\right) \quad\left(\text { or } a_{n-1} a_{n} \Phi_{B}^{s}(\tau)=\Phi_{B}^{s}\left(\eta^{*} \tau\right)\right)
$$

Let $\eta^{*} \tau=D_{0}+D_{1} a_{n}+\cdots$ (or $\eta^{*} \tau=E_{0}+E_{1} a_{n}+E_{2} a_{n-1}+E_{3} a_{n-1} a_{n}+\cdots$ ), where $D_{0}, D_{1}$ (or $E_{0}, E_{1}, E_{2}, E_{3}$ are independent of $a_{p+1}, \ldots, a_{n}$ and the dots denote the linear combination of monomials in $a_{p+1}, \ldots, a_{n}$ other than $1, a_{n}$ (or 1 , $a_{n}, a_{n-1}, a_{n-1} a_{n}$ ) with coefficients from $\mathscr{T}^{(0,2)}\left(\mathbf{R}^{n}\right)$. Let $\tilde{\tau}=D_{0}+D_{1}$ (or $\tilde{\tau}=E_{0}+$ $\left.E_{1}+E_{2}+E_{3}\right)$. Then by the homogeneous function theorem, cf. [6], $\Phi_{B}^{s}(\tau)=$ 
$\Phi_{B}^{s}(\tilde{\tau})$, and similarly for $C$.

It is easy to see that $\tilde{\tau}$ is of the form (4.3) (or $(4.3)^{\prime}$ ). Then using the assumption of the lemma we obtain $\Phi_{B}(\tau)=\Phi_{B}(\tilde{\tau})=\Phi_{C}(\tilde{\tau})=\Phi_{C}(\tau)$.

Lemma 4.3. Let $B \in \mathscr{T}(A, n, 1), n \geq p+2$ (or $B \in \mathscr{T}(A, n, 2), n \geq p+$ 3). Given $t \in \mathbf{R}$ we put $\varphi_{t}=\left(x^{1}, \ldots, x^{p}, t x^{p+1}, x^{p+2}, \ldots, x^{n}\right): \mathbf{R}^{n} \rightarrow \mathbf{R}^{n}$. Let $\rho \in$ $\mathscr{T}^{(0,2)}\left(\mathbf{R}^{n}\right)$ be such that $\varphi_{0}^{*} \rho=0$. Then $\Phi_{B}(\tau)=\Phi_{B}(\tau+\rho)$ for any $\tau \in \mathscr{T}^{(0,2)}\left(\mathbf{R}^{n}\right)$.

Proof of Lemma 4.3. We see that for $t \neq 0$ the diffeomorphism $\varphi_{t}$ preserves $e$ and $\partial_{n}$ (or $e, \partial_{n-1}, \partial_{n}$ ). Using the invariancy of $B$ with respect to $\varphi_{t}, t \neq 0$, we obtain $\Phi_{B}(\tau)=\Phi_{B}\left(\varphi_{t}^{*} \tau\right)$. If $t \rightarrow 0$, then $\Phi_{B}(\tau)=\Phi_{B}\left(\varphi_{0}^{*} \tau\right)$. Since $\varphi_{0}^{*} \rho=0$, then $\varphi_{0}^{*}(\tau+\rho)=\varphi_{0}^{*} \tau$. Hence $\Phi_{B}(\tau)=\Phi_{B}\left(\varphi_{0}^{*} \tau\right)=\Phi_{B}\left(\varphi_{0}^{*}(\tau+\rho)\right)=\Phi_{B}(\tau+\rho)$.

Lemma 4.4. Let $B, C \in \mathscr{T}(A, n, 2), n \geq p+3$. If $\Phi_{B}(\tau)=\Phi_{C}(\tau)$ for any $\tau \in \mathscr{T}^{(0,2)}\left(\mathbf{R}^{n}\right)$ of the form

$$
\begin{aligned}
\tau & =q^{*} \tau_{0}+x^{n} q^{*} \tau_{1}+x^{n-1} q^{*} \tau_{2}+\left(q^{*} \omega_{0}\right) \otimes d x^{n} \\
& +d x^{n} \otimes\left(q^{*} \omega_{1}\right)+\left(q^{*} \omega_{2}\right) \otimes d x^{n-1}+d x^{n-1} \otimes\left(q^{*} \omega_{3}\right) \\
& +x^{n}\left(q^{*} \omega_{4}\right) \otimes d x^{n-1}-x^{n-1}\left(q^{*} \omega_{4}\right) \otimes d x^{n} \\
& +x^{n} d x^{n-1} \otimes\left(q^{*} \omega_{5}\right)-x^{n-1} d x^{n} \otimes\left(q^{*} \omega_{5}\right) \\
& +f_{0}\left(x^{1}, \ldots, x^{p}\right) d x^{n-1} \otimes d x^{n}+f_{1}\left(x^{1}, \ldots, x^{p}\right) d x^{n} \otimes d x^{n-1}
\end{aligned}
$$

where $\tau_{0}, \tau_{1}, \tau_{2} \in \mathscr{T}^{(0,2)}\left(\mathbf{R}^{p}\right), \omega_{0}, \ldots, \omega_{5} \in \mathscr{T}^{(0,1)}\left(\mathbf{R}^{p}\right), f_{0}, f_{1}: \mathbf{R}^{p} \rightarrow \boldsymbol{R}$ and $q: \mathbf{R}^{n}$ $=\mathbf{R}^{p} \times \mathbf{R}^{n-p} \rightarrow \mathbf{R}^{p}$ is the projection, then $B=C$.

Proof of Lemma 4.4. Let $\tau_{0}, \ldots, \tau_{3} \in \mathscr{T}^{(0,2)}\left(\mathbf{R}^{p}\right), \omega_{0}, \ldots, \omega_{7} \in \mathscr{T}^{(0,1)}\left(\mathbf{R}^{p}\right)$ and $f_{0}, f_{1}: \mathbf{R}^{p} \rightarrow \mathbf{R}$. Let $\tilde{\tau}$ be given by $(4.3)^{\prime}$. By Lemma 4.2 it is sufficient to show that $\Phi_{B}(\tilde{\tau})=\Phi_{C}(\tilde{\tau})$.

Let $\tau$ be given by (4.6) with $\tau_{0}, \tau_{1}, \tau_{2}, \omega_{0}, \omega_{1}, \omega_{2}, \omega_{3}, f_{0}, f_{1}$ as above and with $\frac{\omega_{4}-\omega_{5}}{2}$ and $\frac{\omega_{6}-\omega_{7}}{2}$ playing the role of $\omega_{4}$ and $\omega_{5}$ respectively. Then by the assumption, $\Phi_{B}(\tau)=\Phi_{C}(\tau)$.

The diffeomorphism $\beta=\left(x^{1}, \ldots, x^{p}, x^{p+1}+x^{n-1} x^{n}, x^{p+1}, \ldots, x^{n}\right): \mathbf{R}^{n} \rightarrow$ $\mathbf{R}^{n}$ preserves $\left(T^{A} \partial_{n-1}(e), T^{A} \partial_{n}(e)\right)=j^{\bar{A}}\left(t^{1}, \ldots, t^{p}, 0, \ldots, 0, t, \bar{t}\right)$, where $\bar{A}=$ $C_{0}^{\infty}\left(\mathbf{R}^{p} \times \mathbf{R}^{2}\right) /\left\langle\underline{A},\left(\bar{t}^{2}, \bar{t} t,(t)^{2}\right\rangle\right.$.

Let $\omega^{\prime}=q^{*}\left(\frac{\omega_{5}+\omega_{4}}{2}\right)$ and $\omega^{\prime \prime}=q^{*}\left(\frac{\omega_{7}+\omega_{6}}{2}\right)$. Using Lemma 4.3 with $\rho=$ $x^{p+1} q^{*} \tau_{3}+\omega^{\prime} \otimes d x^{p+1}+d x^{p+1} \otimes \omega^{\prime \prime}$ and the invariancy of $B$ with respect to $\beta$ 
we have

$$
\begin{aligned}
\Phi_{B}(\tau)= & \Phi_{B}\left(\tau+x^{p+1} q^{*} \tau_{3}+\omega^{\prime} \otimes d x^{p+1}+d x^{p+1} \otimes \omega^{\prime \prime}\right) \\
= & \Phi_{B}\left(\beta^{*}\left(\tau+x^{p+1} q^{*} \tau_{3}+\omega^{\prime} \otimes d x^{p+1}+d x^{p+1} \otimes \omega^{\prime \prime}\right)\right) \\
= & \Phi_{B}\left(\tau+x^{p+1} q^{*} \tau_{3}+\omega^{\prime} \otimes d x^{p+1}+d x^{p+1} \otimes \omega^{\prime \prime}\right. \\
& \quad+x^{n-1} x^{n} q^{*} \tau_{3}+x^{n} \omega^{\prime} \otimes d x^{n-1}+x^{n-1} \omega^{\prime} \otimes d x^{n} \\
& \left.\quad+x^{n} d x^{n-1} \otimes \omega^{\prime \prime}+x^{n-1} d x^{n} \otimes \omega^{\prime \prime}\right) \\
= & \Phi_{B}\left(\tau+x^{n-1} x^{n} q^{*} \tau_{3}+x^{n} \omega^{\prime} \otimes d x^{n-1}+x^{n-1} \omega^{\prime} \otimes d x^{n}\right. \\
& \left.\quad+x^{n} d x^{n-1} \otimes \omega^{\prime \prime}+x^{n-1} d x^{n-1} \otimes \omega^{\prime \prime}\right)=\Phi_{B}(\tilde{\tau})
\end{aligned}
$$

and similarly for $C$. Hence $\Phi_{B}(\tilde{\tau})=\Phi_{C}(\tilde{\tau})$, as well.

Lemma 4.5. Let $B \in \mathscr{T}(A, n, 1), n \geq p+2$ (or $B \in \mathscr{T}(A, n, 2), n \geq p+3$ ). Suppose that $\tau_{0}, \tau_{1}, \bar{\tau}_{0}, \bar{\tau}_{1} \in \mathcal{T}^{(0,2)}\left(\mathbf{R}^{p}\right), \omega_{0}, \omega_{1}, \bar{\omega}_{0}, \bar{\omega}_{1} \in \mathscr{T}^{(0,1)}\left(\mathbf{R}^{p}\right)\left(\right.$ or $\tau_{0}, \tau_{1}, \tau_{2}$, $\bar{\tau}_{0}, \bar{\tau}_{1}, \bar{\tau}_{2} \in \mathscr{T}^{(0,2)}\left(\mathbf{R}^{p}\right), \omega_{0}, \ldots, \omega_{5}, \bar{\omega}_{0}, \ldots, \bar{\omega}_{5} \in \mathscr{T}^{(0,1)}\left(\mathbf{R}^{p}\right), f_{0}, f_{1}, \bar{f}_{0}, \bar{f}_{1}: \mathbf{R}^{p} \rightarrow$ R) are such that:

$$
\begin{gathered}
{\left[\left[\tau_{\alpha}\right]\right]_{A}=\left[\left[\bar{\tau}_{\alpha}\right]\right]_{A} \text { for } \alpha=0,1 \text { and }\left[\omega_{\beta}\right]_{A}=\left[\bar{\omega}_{\beta}\right]_{A} \text { for } \beta=0,1} \\
\operatorname{or}\left[\left[\tau_{\alpha}\right]\right]_{A}=\left[\left[\bar{\tau}_{\alpha}\right]\right]_{A} \text { for } \alpha=0,1,2,\left[\omega_{\beta}\right]_{A}=\left[\bar{\omega}_{\beta}\right]_{A} \text { for } \beta=0, \ldots, 5, \\
\text { and } \left.j^{A} f_{\gamma}=j^{A} \bar{f}_{\gamma} \text { for } \gamma=0,1\right) .
\end{gathered}
$$

Let $\tau$ be equal to the right side of (4.3) (or (4.6)) and $\bar{\tau}$ be equal to the right side of (4.3) (or (4.6)) with $\tau_{0}, \ldots$ replaced by $\bar{\tau}_{0}, \ldots$ Then $\Phi_{B}(\tau)=\Phi_{B}(\bar{\tau})$.

Proof of Lemma 4.5. Let $\tilde{\tau}$ be as in (4.3) (or 4.3)'). Let $F: \mathbf{R}^{p} \rightarrow \boldsymbol{R}$ be a map in $x^{1}, \ldots, x^{p}$ and $\eta: \mathbf{R}^{p} \rightarrow \mathbf{R}$ be a map in $x^{1}, \ldots, x^{p}$ such that $\operatorname{germ}_{0}\left(\eta\left(t^{1}, \ldots\right.\right.$, $\left.\left.t^{p}\right)\right) \in \underline{A}$. Reminding the definitions of $[[.]]_{A},[.]_{A}$ and $j^{A}($.) (see Section 1) and using Lemma 4.4 it is sufficient to show that

$$
\Phi_{B}(\tilde{\tau})=\Phi_{B}\left(\tilde{\tau}+F X^{0} X^{1} X^{2} d X^{3} \otimes d X^{4}\right),
$$

where $X^{0}, X^{1}, X^{2}, X^{3}, X^{4} \in\left\{1, \eta\left(x^{1}, \ldots, x^{p}\right), x^{1}, \ldots, x^{p}, x^{n}\right\}$ (or $X^{0}, X^{1}, X^{2}$, $\left.X^{3}, X^{4} \in\left\{1, \eta\left(x^{1}, \ldots, x^{p}\right), x^{1}, \ldots, x^{p}, x^{n-1}, x^{n}\right\}\right)$ and $\operatorname{card}\left(\left\{\alpha: X^{\alpha}=\eta\right\}\right)=1$.

We put $\tilde{X}^{\alpha}=x^{p+1}$ if $X^{\alpha}=\eta$ and $\tilde{X}^{\alpha}=X^{\alpha}$ otherwise. The diffeomorphism $\beta$ $=\left(x^{1}, \ldots, x^{p}, x^{p+1}+\eta\left(x^{1}, \ldots, x^{p}\right), x^{p+2}, \ldots, x^{n}\right.$ ) preserves $e$ and $\partial_{n}$ (or $e, \partial_{n-1}$ and $\partial_{n}$ ). Then using Lemma 4.3 with $\rho=F \tilde{X}^{0} \tilde{X}^{1} \tilde{X}^{2} d \tilde{X}^{3} \otimes d \tilde{X}^{4}$ and the invariancy of $B$ with respect to $\beta$ we have

$$
\Phi_{B}(\tilde{\tau})=\Phi_{B}\left(\tilde{\tau}+F \tilde{X}^{0} \tilde{X}^{1} \tilde{X}^{2} d \tilde{X}^{3} d \tilde{X}^{4}\right)=\Phi_{B}\left(\beta^{*}\left(\tilde{\tau}+F \tilde{X}^{0} \tilde{X}^{1} \tilde{X}^{2} d \tilde{X}^{3} d \tilde{X}^{4}\right)\right)
$$




$$
\begin{aligned}
& =\Phi_{B}\left(\tilde{\tau}+F \tilde{X}^{0} \tilde{X}^{1} \tilde{X}^{2} d \tilde{X}^{3} d \tilde{X}^{4}+F X^{0} X^{1} X^{2} d X^{3} d X^{4}\right) \\
& =\Phi_{B}\left(\tilde{\tau}+F X^{0} X^{1} X^{2} d X^{3} d X^{4}\right)
\end{aligned}
$$

as well.

From Lemmas $4.2,4.4$ and 4.5 we obtain

Proposition 4.1. Let $A=C_{0}^{\infty}\left(\mathbf{R}^{p}\right) / \underline{A}$ be a fixed Weil algebra. Let $n \geq p+2$ (or $n \geq p+3$ ) be a fixed natural number. For any $B \in \mathscr{T}(A, n, 1)$ (or $B \in \mathscr{T}(A, n, 2)$ ) define $G_{B}:\left(S^{A}\right)^{2} \times\left(Q^{A}\right)^{2}=S^{A} \times S^{A} \times Q^{A} \times Q^{A} \rightarrow \mathbf{R}\left(\right.$ or $G_{B}:\left(S^{A}\right)^{3} \times\left(Q^{A}\right)^{5} \times$ $\left.\left(T^{A} \mathbf{R}\right)^{2} \rightarrow \mathbf{R}\right)$ by

$$
\begin{gathered}
G_{B}\left(\left[\left[\tau_{0}\right]\right]_{A},\left[\left[\tau_{1}\right]\right]_{A},\left[\omega_{0}\right]_{A},\left[\omega_{1}\right]_{A}\right)=\Phi_{B}(\tilde{\tau}) \\
\left(\operatorname{or~} G_{B}\left(\left[\left[\tau_{0}\right]\right]_{A},\left[\left[\tau_{1}\right]\right]_{A},\left[\left[\tau_{2}\right]\right]_{A},\left[\omega_{0}\right]_{A}, \ldots,\left[\omega_{5}\right]_{A}, j^{A} f_{0}, j^{A} f_{1}\right)=\Phi_{B}(\tau)\right),
\end{gathered}
$$

where $\tilde{\tau}$ is given by (4.3) (or $\tau$ is given by (4.6)). Then the function

$$
\begin{gathered}
G: \mathscr{T}(A, n, 1) \rightarrow C^{\infty}\left(\left(S^{A}\right)^{2} \times\left(Q^{A}\right)^{2}\right), \quad G(B)=G_{B} \\
\left(\text { or } G: \mathscr{T}(A, n, 2) \rightarrow C^{\infty}\left(\left(S^{A}\right)^{3} \times\left(Q^{A}\right)^{5} \times\left(T^{A} \mathbf{R}\right)^{2}\right), \quad G(B)=G_{B}\right)
\end{gathered}
$$

is a monomorphism of $C^{\infty}\left(S^{A}\right)$-modules, provided the $C^{\infty}\left(S^{A}\right)$-module structure in $C^{\infty}\left(\left(S^{A}\right)^{2} \times\left(Q^{A}\right)^{2}\right)$ (or in $\left.C^{\infty}\left(\left(S^{A}\right)^{3} \times\left(Q^{A}\right)^{5} \times\left(T^{A} \mathbf{R}\right)^{2}\right)\right)$ is given by $(\lambda f)\left(a_{0}, a_{1}\right.$, $\left.b_{0}, b_{1}\right)=\lambda\left(a_{0}\right) f\left(a_{0}, a_{1}, b_{0}, b_{1}\right) \quad\left(\right.$ or $\quad(\lambda f)\left(a_{0}, \ldots, a_{2}, b_{0}, \ldots, b_{5}, c_{0}, c_{1}\right)=\lambda\left(a_{0}\right)$ $\left.f\left(a_{0}, \ldots, a_{2}, b_{0}, \ldots, b_{5}, c_{0}, c_{1}\right)\right)$, where $\lambda \in C^{\infty}\left(S^{A}\right), f \in C^{\infty}\left(\left(S^{A}\right)^{2} \times\left(Q^{A}\right)^{2}\right)$ (or $f \in C^{\infty}\left(\left(S^{A}\right)^{3} \times\left(Q^{A}\right)^{5} \times\left(T^{A} \mathbf{R}\right)^{2}\right)$ ) and $\left(a_{0}, a_{1}, b_{0}, b_{1}\right) \in\left(S^{A}\right)^{2} \times\left(Q^{A}\right)^{2}$ (or $\left.\left(a_{0}, a_{1}, a_{2}, b_{0}, \ldots, b_{5}, c_{0}, c_{1}\right) \in\left(S^{A}\right)^{3} \times\left(Q^{A}\right)^{5} \times\left(T^{A} \mathbf{R}\right)^{2}\right)$.

Pooof. By Lemma 4.5, $G_{B}$ is well-defined. From Lemma 4.2 (or Lemma 4.4) it follows that $G$ is injective. Reminding the definitions of the module structures it is easy to verify that $G$ is a homomorphism of $C^{\infty}\left(S^{A}\right)$-modules

\section{Proof of Theorems 3.1 and 3.2}

Let $B \in \mathscr{T}(A, n, 1)$ (or $B \in \mathscr{T}(A, n, 2)$ ). From the invariancy of $B$ with respect to $\left(x^{1}, \ldots, x^{n-1}, t x^{n}\right)$ preserving $e$ and sending $\partial_{n}$ into $t \partial_{n}$ (or with respect to $\left(x^{1}, \ldots, x^{n-2}, \bar{t} x^{n-1}, t x^{n}\right)$ preserving $e$ and sending $\partial_{n-1}, \partial_{n}$ into $\left.\bar{t} \partial_{n-1}, t \partial_{n}\right)$ it follows that

$$
\begin{gathered}
t G_{B}\left(a_{0}, a_{1}, b_{0}, b_{1}\right)=G_{B}\left(a_{0}, t a_{1}, t b_{0}, t b_{1}\right) \\
\text { (or } \bar{t} G_{B}\left(a_{0}, a_{1}, a_{2}, b_{0}, \ldots, b_{5}, c_{0}, c_{1}\right)
\end{gathered}
$$




$$
\left.=G_{B}\left(a_{0}, t a_{1}, \bar{t} a_{2}, t b_{0}, t b_{1}, \bar{t} b_{2}, \bar{t} b_{3}, \bar{t} t b_{4}, \bar{t} t b_{5}, \bar{t} t c_{0}, \bar{t} t c_{1}\right)\right)
$$

for any $\left(a_{0}, a_{1}, b_{0}, b_{1}\right) \in\left(S^{A}\right)^{2} \times\left(Q^{A}\right)^{2}$ and any $t \in \mathbf{R}-\{0\}$ or for any $\left(a_{0}, a_{1}\right.$, $\left.a_{2}, b_{0}, \ldots, b_{5}, c_{0}, c_{1}\right) \in\left(S^{A}\right)^{3} \times\left(Q^{A}\right)^{5} \times\left(T^{A} \mathbf{R}\right)^{2}$ and any $\left.\bar{t}, t \in \mathbf{R}-\{0\}\right)$. We fix bases in $S^{A}$ and $Q^{A}$ (or in $S^{A}, Q^{A}$ and $T^{A} \mathbf{R}$ ). By the homogeneous function theorem, cf. [6], $G_{B}$ is a linear combination of the coordinates of $a_{1}, b_{0}, b_{1}$ (or a linear combination of the coordinates of $b_{4}, b_{5}, c_{0}, c_{1}, a_{1} \otimes a_{2}, a_{1} \otimes b_{2}, a_{1} \otimes b_{3}, a_{2}$ $\otimes b_{0}, a_{2} \otimes b_{1}, b_{0} \otimes b_{2}, b_{0} \otimes b_{3}, b_{1} \otimes b_{2}, b_{1} \otimes b_{3}$ ) with respect to the bases with coefficients being $C^{\infty}$-maps depending on $a_{0}$. Thus owing to Proposition 4.1 we see that Theorem 3.1 (or Theorem 3.2) will be proved after proving that:

$$
\begin{gathered}
G_{B^{\langle\psi\rangle}}(a, b)=\phi\left(a_{1}\right), \\
G_{B^{\langle\varphi\rangle_{2}}}(a, b)=\varphi\left(b_{0}\right), \quad G_{B^{\langle\varphi\rangle_{1}}}(a, b)=\varphi\left(b_{1}\right)
\end{gathered}
$$

for any $(a, b)=\left(a_{0}, a_{1}, b_{0}, b_{1}\right) \in\left(S^{A}\right)^{2} \times\left(Q^{A}\right)^{2}$, any $\varphi \in\left(Q^{A}\right)^{*}$ and any $\phi \in$ $\left(S^{A}\right)^{*}$ (or after proving that:

$$
\begin{aligned}
& G_{d B^{\langle\varphi\rangle_{2}}}(a, b, c)=-2 \varphi\left(b_{4}\right), \quad G_{d B^{\langle\varphi\rangle_{1}}}(a, b, c)=-2 \varphi\left(b_{5}\right), \\
& G_{B^{\langle\lambda\rangle_{1}}}(a, b, c)=\lambda\left(c_{0}\right), \quad G_{B^{\langle\lambda\rangle_{2}}}(a, b, c)=\lambda\left(c_{1}\right), \\
& G_{B^{\langle\psi\rangle} \otimes B^{\langle\phi\rangle}}(a, b, c)=\phi\left(a_{2}\right) \bar{\psi}\left(a_{1}\right), G_{B^{\langle\varphi\rangle} 2 \otimes B^{\langle\psi\rangle}}(a, b, c)=\varphi\left(b_{2}\right) \psi\left(a_{1}\right), \\
& G_{B^{\langle\varphi\rangle} 1 \otimes B^{\langle\psi\rangle}}(a, b, c)=\varphi\left(b_{3}\right) \psi\left(a_{1}\right), G_{B^{\langle\psi\rangle} \otimes B^{\langle\varphi\rangle_{2}}}(a, b, c)=\psi\left(a_{2}\right) \varphi\left(b_{0}\right), \\
& G_{B^{\langle\phi\rangle} \otimes B^{\langle\varphi\rangle_{1}}}(a, b, c)=\phi\left(a_{2}\right) \varphi\left(b_{1}\right), G_{\left.B^{\langle\varphi\rangle_{2}} \otimes B^{\langle\varphi}\right\rangle_{2}}(a, b, c)=\varphi\left(b_{2}\right) \bar{\varphi}\left(b_{0}\right) \text {, } \\
& G_{B^{\langle\varphi\rangle_{1}} \otimes B}(\varphi\rangle_{2}(a, b, c)=\varphi\left(b_{3}\right) \bar{\varphi}\left(b_{0}\right), G_{B^{\langle\varphi\rangle_{2}} \otimes B^{\langle\varphi\rangle_{1}}}(a, b, c)=\varphi\left(b_{2}\right) \bar{\varphi}\left(b_{1}\right) \text {, } \\
& G_{\left.B^{\langle\varphi\rangle}\right\rangle_{1} \otimes B^{\langle\varphi\rangle_{1}}}(a, b, c)=\varphi\left(b_{3}\right) \bar{\varphi}\left(b_{1}\right)
\end{aligned}
$$

for any $(a, b, c)=\left(a_{0}, a_{1}, a_{2}, b_{0}, \ldots, b_{5}, c_{0}, c_{1}\right) \in\left(S^{A}\right)^{3} \times\left(Q^{A}\right)^{5} \times\left(T^{A} \mathbf{R}\right)^{2}$, any $\lambda \in A^{*}=\left(T^{A} \mathbf{R}\right)^{*}$, any $\varphi, \bar{\varphi} \in\left(Q^{A}\right)^{*}$ and any $\left.\phi, \bar{\phi} \in\left(S^{A}\right)^{*}\right)$.

We prove (5.1)-(5.5) as follows.

Let $\varphi \in\left(Q^{A}\right)^{*}, \phi \in\left(S^{A}\right)^{*}$ and $\lambda \in A^{*}$. Let $\tau_{0}, \tau_{1}, \tau_{2} \in \mathscr{T}^{(0,2)}\left(\mathbf{R}^{p}\right), \omega_{0}, \ldots$, $\omega_{5} \in \mathscr{T}^{(0,1)}\left(\mathbf{R}^{\not}\right)$ and $f_{0}, f_{1}: \mathbf{R}^{p} \rightarrow \mathbf{R}$. Let $\tilde{\tau}, \tau$ be given by (4.3) and (4.6) respectively. Let $\tilde{A}=C_{0}^{\infty}\left(\mathbf{R}^{p} \times \mathbf{R}\right) /\left\langle\underline{A},(t)^{2}\right\rangle$. Then $T^{\tilde{A}} M=T T^{A} M$. We observe that given $\alpha \in \mathbf{R}$ we have (see Examples 1.1, 1.2, 1.3, 2.1, 2.2, 2.3 and 2.4 for the notation) 


$$
\begin{aligned}
\left\langle B_{\mathbf{R}^{n}}^{\langle\varphi\rangle_{1}}(\tau), j^{\tilde{A}}\left(t^{1}, \ldots, t^{p}, 0, \ldots, 0, \alpha, t\right)\right\rangle & \\
= & \varphi\left(\left[i^{*}\left(C_{1}^{1}\left(\left(t^{1}, \ldots, t^{p}, 0, \ldots, 0, \alpha, t\right)^{*} \tau \otimes \frac{\partial}{\partial t}\right)\right)\right]_{A}\right) \\
= & \varphi\left(\left[i ^ { * } \left(C _ { 1 } ^ { 1 } \left(\left(\tau_{0}+t \tau^{1}+\alpha \tau_{2}+\omega_{0} \otimes d t+d t \otimes \omega_{1}\right.\right.\right.\right.\right. \\
& \left.\left.\left.\left.\left.\quad-\alpha \omega_{4} \otimes d t-\alpha d t \otimes \omega_{5}\right) \otimes \frac{\partial}{\partial t}\right)\right)\right]_{A}\right)=\varphi\left(\left[\omega_{1}\right]_{A}\right)-\alpha \varphi\left(\left[\omega_{5}\right]_{A}\right)
\end{aligned}
$$

as $\tau_{0}, \tau_{1}, \tau_{2}, \omega_{0}, \omega_{4}$ are independent of $d t$. It follows from (5.6) and the invariancy of $B$ with respect to the diffeomorphism permuting $x^{n-1}$ and $x^{n}$ that

$$
\left\langle B_{\mathbf{R}^{n}}^{\langle\rangle_{1}}(\tau), j^{\tilde{A}}\left(t^{1}, \ldots, t^{p}, 0, \ldots, 0, t, \alpha\right)\right\rangle=\varphi\left(\left[\omega_{3}\right]_{A}\right)+\alpha \varphi\left(\left[\omega_{5}\right]_{A}\right) .
$$

Since $B_{\mathbf{R}^{n}}^{\langle\varphi\rangle_{2}}(\tau)=B_{\mathbf{R}^{n}}^{\langle\varphi\rangle_{1}}\left(\tau^{s}\right)$, it follows from (5.6) and (5.7) that

$$
\begin{aligned}
& \left\langle B_{\mathbf{R}^{n}}^{\langle\varphi\rangle_{2}}(\tau), j^{\tilde{A}}\left(t^{1}, \ldots, t^{p}, 0, \ldots, 0, \alpha, t\right)\right\rangle=\varphi\left(\left[\omega_{0}\right]_{A}\right)-\alpha \varphi\left(\left[\omega_{4}\right]_{A}\right) . \\
& \left\langle B_{\mathbf{R}^{n}}^{\langle\varphi\rangle_{2}}(\tau), j^{\tilde{A}}\left(t^{1}, \ldots, t^{p}, 0, \ldots, 0, t, \alpha\right)\right\rangle=\varphi\left(\left[\omega_{2}\right]_{A}\right)+\alpha \varphi\left(\left[\omega_{4}\right]_{A}\right),
\end{aligned}
$$

We see that

$$
\begin{aligned}
\left\langle B_{\mathbf{R}^{n}}^{\langle\psi\rangle}(\tau), T^{A} \partial_{n}(e)\right\rangle & =\left\langle d\left(\tau^{[\psi]}\right), T^{A} \partial_{n}(e)\right\rangle \\
& =\left.\frac{d}{d t}\right|_{t=0}\left(\tau^{[\psi]}\left(j^{A}\left(t^{1}, \ldots, t^{p}, 0, \ldots, 0, t\right)\right)\right) \\
& =\frac{d}{\left.d t\right|_{t=0}}\left(\phi\left(\left[\left[\left(t^{1}, \ldots, t^{p}, 0, \ldots, 0, t\right)^{*} \tau\right]\right]_{A}\right)\right) \\
& =\frac{d}{\left.d t\right|_{t=0}}\left(\phi\left(\left[\left[\tau_{0}+t \tau_{1}\right]\right]_{A}\right)\right)=\phi\left(\left[\left[\tau_{1}\right]\right]_{A}\right) .
\end{aligned}
$$

From (5.10) and the invariancy of $B^{\langle\psi\rangle}$ with respect to the diffeomorphism permuting $x^{n}$ and $x^{n-1}$ it follows that

$$
\left\langle B_{\mathbf{R}^{n}}^{\langle\psi\rangle}(\tau), T^{A} \partial_{n-1}(e)\right\rangle=\phi\left(\left[\left[\tau_{2}\right]\right]_{A}\right) .
$$

From (5.10) with $\tau_{2}=0, \omega_{2}=\cdots=\omega_{5}=0$ and $f_{0}=f_{1}=0$ it follows (5.1).

From formula (5.6) and (5.8) with $\alpha=0, \tau_{2}=0, \omega_{2}=\cdots=\omega_{5}=0$ and $f_{0}$ $=f_{1}=0$ we obtain formula $(5.2)$.

Since $\left[T^{A} \partial_{n-1}, T^{A} \partial_{n}\right]=0$, it follows from (5.6)-(5.8) that

$$
\begin{aligned}
G_{d B^{\langle\varphi\rangle} 1}\left(\left[\left[\tau_{0}\right]\right]_{A},\left[\left[\tau_{1}\right]\right]_{A},\left[\left[\tau_{2}\right]\right]_{A},\left[\omega_{0}\right]_{A}, \ldots,\left[\omega_{5}\right]_{A}, j^{A} f_{0}, j^{A} f_{1}\right) \\
\quad=\left\langle d\left(B_{\mathbf{R}^{n}}^{\langle\varphi\rangle_{1}}(\tau)\right),\left(T^{A} \partial_{n-1}(e)\right) \otimes\left(T^{A} \partial_{n}(e)\right)\right\rangle \\
\quad=T^{A} \partial_{n-1}(e)\left(\left\langle B_{\mathbf{R}^{n}}^{\langle\varphi\rangle_{1}}(\tau), T^{A} \partial_{n}\right\rangle\right)-T^{A} \partial_{n}(e)\left(\left\langle B_{\mathbf{R}^{n}}^{\langle\varphi\rangle_{1}}(\tau), T^{A} \partial_{n-1}\right\rangle\right)
\end{aligned}
$$




$$
\begin{aligned}
& =\frac{d}{d \alpha \mid \alpha=0}\left(\left\langle B_{\mathbf{R}^{n}}^{\langle\varphi\rangle_{1}}(\tau), j^{\tilde{A}}\left(t^{1}, \ldots, t^{p}, 0, \ldots, 0, \alpha, t\right)\right\rangle\right) \\
& \quad-\frac{d}{d \alpha \mid \alpha=0}\left(\left\langle B_{\mathbf{R}^{n}}^{\langle\varphi\rangle_{1}}(\tau), j^{\tilde{A}}\left(t^{1}, \ldots, t^{p}, 0, \ldots, 0, t, \alpha\right)\right\rangle\right)=-2 \varphi\left(\left[\omega_{5}\right]_{A}\right)
\end{aligned}
$$

and (similarly)

$$
G_{d B^{\langle\varphi\rangle_{2}}}\left(\left[\left[\tau_{0}\right]\right]_{A},\left[\left[\tau_{1}\right]\right]_{A},\left[\left[\tau_{2}\right]\right]_{A},\left[\omega_{0}\right]_{A}, \ldots,\left[\omega_{5}\right]_{A}, j^{A} f_{0}, j^{A} f_{1}\right)=-2 \varphi\left(\left[\omega_{4}\right]_{A}\right) .
$$

Therefore formulas (5.3) are proved.

We see that $G_{B \otimes \bar{B}}\left(\left[\left[\tau_{0}\right]\right]_{A},\left[\left[\tau_{1}\right]\right]_{A},\left[\left[\tau_{2}\right]\right]_{A},\left[\omega_{0}\right]_{A}, \ldots,\left[\omega_{5}\right]_{A}, j^{A} f_{0}, j^{A} f_{1}\right)=$ $\left\langle B_{\mathbf{R}^{n}}(\tau) \otimes \bar{B}_{\mathbf{R}^{n}}(\tau),\left(T^{A} \partial_{n-1}(e)\right) \otimes\left(T^{A} \partial_{n}(e)\right)\right\rangle$ for any $B, \bar{B} \in \mathscr{T}(A, n, 1)$. We know that $T^{A} \partial_{n}(e)=j^{\tilde{A}}\left(t^{1}, \ldots, t^{p}, 0, \ldots, 0, t\right)$ and $T^{A} \partial_{n-1}(e)=j^{\tilde{A}}\left(t^{1}, \ldots, t^{p}, 0\right.$, $\ldots, 0, t, 0)$. Therefore formulas $(5.5)$ are clear because of $(5.10),(5.11)$ and (5.6)-(5.9) with $\alpha=0$.

It remains to show formulas (5.4). We have

$$
\begin{aligned}
G_{B^{\langle\lambda\rangle_{1}}}\left(\left[\left[\tau_{0}\right]\right]_{A},\left[\left[\tau_{1}\right]\right]_{A},\left[\left[\tau_{2}\right]\right]_{A},\left[\omega_{0}\right]_{A}, \ldots,\left[\omega_{5}\right]_{A}, j^{A} f_{0}, j^{A} f_{1}\right) \\
\quad=\left\langle B_{\mathbf{R}^{n}}^{\langle\lambda\rangle_{1}}(\tau),\left(T^{A} \partial_{n-1}(e)\right) \otimes\left(T^{A} \partial_{n}(e)\right)\right\rangle \\
\quad=\left\langle\lambda \circ T^{A}(\tau) \circ(I \times I),\left(T^{A} \partial_{n-1}(e)\right) \otimes\left(T^{A} \partial_{n}(e)\right)\right\rangle \\
\quad=\left(\lambda \circ T^{A}\left(\left\langle\tau, \partial_{n-1} \otimes \partial_{n}\right\rangle\right)\right)(e)=\left(\lambda \circ T^{A} f_{0}\right)(e)=\lambda\left(j^{A} f_{0}\right) .
\end{aligned}
$$

The first formula of (5.4) is proved. Since $B_{\mathbf{R}^{n}}^{\langle\lambda\rangle_{2}}(\tau)=B_{\mathbf{R}^{n}}^{\langle\lambda\rangle_{1}}\left(\tau^{s}\right)$, the second formula of (5.4) is an immediate consequence of the first one.

\section{Acknowledgements}

This work is supported by a grant of KBN no: 2 P 30103004.

\section{REFERENCES}

[1] M. Doupovec, J. Kurek, Liftings of covariant $(0,2)$ tensor fields to the bundle of $k$-dimensional 1-velocities, Suppl. Rend. Circolo Math. Palermo (in press).

[2] D. B. A. Epstein, Natural tensors on Riemannian manifolds, J. Differential Geom., 10 (1975), 613-645.

[3] J. Gancarzewicz, Liftings of functions and vector fields to natural bundles, Warszawa 1983, Dissertationes Mathematicae CCXII.

[4] J. Gancarzewicz, W. M. Mikulski, Z. Pogoda, Lifts of some tensor fields and connections to product preserving functors, Nagoya Math. J., 135 (1994), 1-41.

[5] I. Kolár̆, On the natural operators on vector fields, Ann. Global Analysis and Geometry, 6(2) (1988), 109-117.

[6] I. Kolář, P. W. Michor, J. Slovák, Natural operations in differential geometry, 
Springer-Verlag, Berlin, 1993.

[ 7 ] J. Kurek, On the first order natural operators transforming 1-forms on a manifold to linear frame bundle, Demonstratio Math., 26 (1993), 287-293.

[8] W. M. Mikulski, Some natural operations on vector fields, Rend. Math. (Serie VII) 12, Roma (1992), 783-803.

[9] - Some natural constructions on vector fields and higher order cotangent bundles, Mh. Math., 117, (1994), 107-119.

[10] - Natural transformations transforming functions and vector fields to functions on some natural bundles, Math. Bohemica, 117 (1992), 217-223.

[11] - Natural transformations transforming vector fields into affinors on the extended $\boldsymbol{r}$-th order tangent bundles, Arch. Math. Brno, 29 (1993), 59-70.

[12] - Natural liftings of foliations to the tangent bundle, Math. Bohemica, 117 (1992), 409-414.

[13] - The natural operators lifting 1 -forms on manifolds to the bundles of $A$-velocities, Mh. Math., 119 (1995), 63-77.

[14] - Natural base-extending operators of foliations into foliations on the Weil functors, Geom. Dedicata, 54 (1995), 129-136.

[15] A. Morimoto, Prolongations of connections to bundles of infinitely near points, J. Differential Geom., 11 (1976), 479-498.

[16] - Liftings of tensor fields and connections to tangential fibre bundles of higher order, Nagoya Math. J., 40 (1970), 85-97.

[17] A. Weil, Théorie des points proches sur les variétés différentiables, Colloque du C. N. R. S. Strasbourg (1953), 111-117.

Institute of Mathematics

Jagellonian University

Kraków Reymonta 4 (Poland) 